\title{
The magnetic shear-current effect: generation of large-scale magnetic fields by the small-scale dynamo
}

\author{
J. Squire ${ }^{1,2, \dagger}$ and A. Bhattacharjee ${ }^{2,3}$ \\ ${ }^{1}$ TAPIR, Mailcode 350-17, California Institute of Technology, Pasadena, CA 91125, USA \\ ${ }^{2}$ Max Planck/Princeton Center for Plasma Physics, Department of Astrophysical Sciences and Princeton \\ Plasma Physics Laboratory, Princeton University, Princeton, NJ 08543, USA \\ ${ }^{3}$ Department of Astrophysical Sciences and Princeton Plasma Physics Laboratory, Princeton University, \\ Princeton, NJ 08543, USA
}

(Received 8 December 2015; revised 16 February 2016; accepted 18 February 2016)

A novel large-scale dynamo mechanism, the magnetic shear-current effect, is discussed and explored. The effect relies on the interaction of magnetic fluctuations with a mean shear flow, meaning the saturated state of the small-scale dynamo can drive a large-scale dynamo - in some sense the inverse of dynamo quenching. The dynamo is non-helical, with the mean field $\alpha$ coefficient zero, and is caused by the interaction between an off-diagonal component of the turbulent resistivity and the stretching of the large-scale field by shear flow. Following up on previous numerical and analytic work, this paper presents further details of the numerical evidence for the effect, as well as an heuristic description of how magnetic fluctuations can interact with shear flow to produce the required electromotive force. The pressure response of the fluid is fundamental to this mechanism, which helps explain why the magnetic effect is stronger than its kinematic cousin, and the basic idea is related to the well-known lack of turbulent resistivity quenching by magnetic fluctuations. As well as being interesting for its applications to general high Reynolds number astrophysical turbulence, where strong small-scale magnetic fluctuations are expected to be prevalent, the magnetic shear-current effect is a likely candidate for large-scale dynamo in the unstratified regions of ionized accretion disks. Evidence for this is discussed, as well as future research directions and the challenges involved with understanding details of the effect in astrophysically relevant regimes.

\section{Introduction}

Magnetic fields pervade the universe. From the scales of planets up to galaxy clusters and beyond, they are not only ubiquitous but have also proven dramatically important in a wide variety of astrophysical and geophysical processes. Despite this, our understanding the mechanisms that lead to their creation and sustenance is hazy, and improving this remains an outstanding theoretical challenge. Much of the theory of field generation focuses on turbulent dynamo, in which magnetic fields

$\dagger$ Email address for correspondence: jsquire@caltech.edu 
are stretched and twisted by turbulent fluctuations in such a way as to increase their strength, resulting in exponential instability. Through this process, very small seed fields - arising, for example, from the Biermann battery or kinetic instabilities might be amplified enormously by plasma motions to the levels seen throughout the universe today.

Interestingly, magnetic fields are generically observed to be correlated over larger scales than the underlying fluid motions, and such large-scale dynamos are of vital importance for explaining astrophysical fields. The classic mechanism to allow such behaviour is the kinematic $\alpha$ effect* (Moffatt 1978; Krause \& Rädler 1980). Here, the small-scale fluid turbulence interacts with a large-scale magnetic field in such a way that an electromotive force $(\mathrm{EMF}$, represented by $\mathcal{E}$ ) is created in proportion the magnetic field itself $(\mathcal{E} \sim \alpha \boldsymbol{B})$, potentially causing an instability to develop. To allow such behaviour, the turbulence must break statistical symmetry in some way, either through a net helicity or through stratification. However, various problems with large scale $\alpha$ dynamos become apparent when one considers how field growth rates change with the scale of the field - specifically, the smallest scales always grow the most rapidly (Kulsrud \& Anderson 1992; Boldyrev, Cattaneo \& Rosner 2005). In addition, as a consequence of the conservation of magnetic helicity, these small-scale magnetic fields act to decrease the large-scale growth rate in a way that scales very unfavourably to high Reynolds numbers - the problem of 'catastrophic quenching' (Gruzinov \& Diamond 1994; Bhattacharjee \& Yuan 1995; Cattaneo \& Hughes 2009). While a variety of solutions to such problems have been explored, primarily focused on the transport of magnetic helicity (Vishniac \& Cho 2001; Blackman \& Field 2002; Subramanian \& Brandenburg 2004; Ebrahimi \& Bhattacharjee 2014; Tobias \& Cattaneo 2014), the scaling of $\alpha$ dynamos to astrophysically relevant regimes is still far from understood. Such issues are not necessarily confined to the $\alpha$ effect either. Above even moderate Reynolds numbers, the fast-growing small-scale dynamo (field generation on scales at and below that of the fluid turbulence; Schekochihin et al. 2007) implies that velocity fluctuations should always be accompanied by magnetic fluctuations of a similar magnitude (Schekochihin et al. 2004). This challenges the relevance of the classical kinematic dynamo picture (Cattaneo \& Hughes 2009), which focuses purely on the properties of the small-scale velocity fields.

In this paper - as well as in Squire \& Bhattacharjee (2015b) (hereafter Paper I), Squire \& Bhattacharjee (2015c) (hereafter Paper II) and Squire \& Bhattacharjee $(2015 d)$ (hereafter Paper III) - we suggest and explore a new dynamo mechanism in which the small-scale magnetic fluctuations, in combination with a background shear flow, are the primary driver of the large-scale field growth. Termed the 'magnetic shear-current effect', by analogy to earlier kinematic suggestions (Urpin 1999; Rogachevskii \& Kleeorin 2003), the effect is non-helical (the dynamo $\alpha$ coefficient is zero), and is driven by an off-diagonal component of the turbulent resistivity tensor. There are two principal reasons for our interest in this effect. The first is that the mechanism is not an $\alpha$ effect, which implies that the dynamo can operate in turbulence with a high degree of symmetry. This makes it a possible mechanism to explain the dynamo seen in the central regions of accretion disk simulations (Brandenburg et al. 1995; Hawley, Gammie \& Balbus 1996), and we have seen good evidence that this is indeed the case (see $\S 4$, as well as Squire \& Bhattacharjee 2015a). The second reason for our interest in the magnetic shear-current effect stems from the intriguing possibility of a large-scale dynamo being driven by

*The term kinematic denotes the situation where velocity fluctuations are unaffected by the magnetic field. 
the saturated state of the small-scale dynamo. In some sense, this is the inverse of the quenching described in the previous paragraph - the small-scale dynamo, far from quenching large-scale growth, is its primary driver. Such a large-scale dynamo paradigm is far removed from classical kinematic theory, relying on saturation of the small-scale turbulent fields. Accordingly, the magnetic shear-current effect is an inherently nonlinear dynamo mechanism (Tobias, Cattaneo \& Brummell 2011a), although it can be driven by a turbulent velocity field rather than resulting from the nonlinear development of a laminar instability.

Proving the existence and importance of a dynamo instability can be delicate: numerical simulations of turbulence are necessarily noisy, one is limited in available Reynolds numbers (and thus the ability to prove a dynamo will remain active at high values), and when large-scale field growth is observed, it can be difficult to show convincingly that it is not some other (possibly unknown) mechanism that is responsible. These problems are exacerbated in the magnetically driven case studied in this work. In particular, due to the finite size of any numerically realizable mean-field average, the large-scale field will quickly come into equipartition with the turbulent bath of fluctuations, robbing the researcher of the ability to study the dynamo during a long period of exponential growth. In other words, the dynamo will very quickly transition into its saturated state (where the large-scale fields have a strong influence on the small-scale turbulence), exacerbating measurement of the properties of the linear growth phase, or even the observation of its qualitative behaviour. For these reasons, we have attempted to tackle the problem from a variety of different angles, including analytically with the second-order correlation approximation (Paper III), through quasi-linear theory and statistical simulation (Paper II) and using direct numerical simulations (Paper I; Paper II). We also employ the novel technique of using an ensemble of simulations to study the statistics of the mean field without taking time averages. Our hope is that with this variety of methods, which all lead to the same general conclusions, we present convincing evidence for the existence of the magnetic shear-current effect and its potential importance in astrophysical dynamo theory.

The present paper serves two purposes. The first is to give a more heuristic and physical description of the magnetic shear-current effect, which is done throughout $\S 2$. Following a basic description of the mechanism in the language of mean-field dynamo theory, we describe (with diagrams and simple explanations) how magnetic fluctuations, interacting with a large-scale magnetic field and shear flow, can generate the correlated velocity perturbations that are required for a mean-field dynamo instability. Interestingly, we find that the pressure response of the velocity fluctuations is fundamental to the operation of the dynamo, and simple arguments based on the directions of induced perturbations explain qualitatively why one might expect the magnetic effect to be stronger than the kinematic effect. The second purpose of this paper, discussed in $\S 3$, is to expand upon, and provide further details for, the analysis and simulations presented in Paper I. In particular, these simulations demonstrate for the first time (as far as we are aware) that the saturated state of the small-scale dynamo can drive a large-scale dynamo. Our method for showing this involves measuring the transport coefficients before and after small-scale dynamo saturation. This illustrates that strong magnetic fluctuations can decrease, and change the sign of, a particularly important component of the tensorial turbulent resistivity (termed $\eta_{y x}$ throughout the text), in a way that is consistent with observed mean-field evolution. Since the methods used to show this are somewhat non-standard, considerable effort is put into explaining these and ensuring that the coefficients are determined accurately. 
This is done both through direct comparison with standard methods in lower Reynolds number kinematic dynamos (appendix A), and by using the measured coefficients to solve for the expected large-scale field evolution.

Finally, in $\S 4$, we conclude and present a more in-depth discussion of why the magnetic shear-current effect is interesting as a mechanism for large-scale dynamo. This includes some analysis of the evidence for the effect's importance in driving the dynamo in the central regions of accretion disks, which is primarily based on the Prandtl number dependence of its nonlinear saturation (Squire \& Bhattacharjee 2015a).

\section{The physical mechanism for the magnetic shear-current effect}

In this section we describe how homogeneous non-helical magnetic fluctuations, influenced by a large-scale shear flow and magnetic field gradient, can generate an EMF that acts to reinforce the large-scale magnetic field. We shall start by describing the form of the EMF that allows for such behaviour, as well as constraints due to the symmetries of the system, then consider a simplified cartoon picture for how the interaction of magnetic fluctuations with velocity shear and a large-scale field gradient might produce this EMF.

All studies in this work are carried out in the context of the incompressible MHD equations with a background shear flow $\boldsymbol{U}_{0}=-S x \hat{\boldsymbol{y}}$,

$$
\begin{gathered}
\frac{\partial \boldsymbol{U}_{T}}{\partial t}-S x \frac{\partial \boldsymbol{U}_{T}}{\partial y}+\left(\boldsymbol{U}_{T} \cdot \nabla\right) \boldsymbol{U}_{T}+2 \Omega \hat{z} \times \boldsymbol{U}_{T}+\nabla p=S U_{T x} \hat{\boldsymbol{y}}+\boldsymbol{B}_{T} \cdot \nabla \boldsymbol{B}_{T}+\bar{v} \nabla^{2} \boldsymbol{U}_{T}+\boldsymbol{\sigma}_{\boldsymbol{u}}, \\
\frac{\partial \boldsymbol{B}_{T}}{\partial t}-S x \frac{\partial \boldsymbol{B}_{T}}{\partial y}=-S B_{T x} \hat{\boldsymbol{y}}+\nabla \times\left(\boldsymbol{U}_{T} \times \boldsymbol{B}_{T}\right)+\bar{\eta} \nabla^{2} \boldsymbol{B}_{T}, \\
\nabla \cdot \boldsymbol{U}_{T}=0, \quad \nabla \cdot \boldsymbol{B}_{T}=0 .
\end{gathered}
$$

Here $\Omega$ is a mean rotation of the frame, and $\bar{v}$ and $\bar{\eta}$ are the normalized viscosity and resistivity, respectively. Since all quantities are normalized to one it is convenient to define $R e=1 / \bar{v}$ and $R m=1 / \bar{\eta}$ for the Reynolds and magnetic Reynolds number, and their ratio is the Prandtl number $P m=R m / R e . \sigma_{u}$ denotes a non-helical driving noise source, white in time, which can be used to generate an homogenous bath of small-scale velocity fluctuations. $\boldsymbol{U}_{T}$ and $\boldsymbol{B}_{T}$ in (2.1) are simply the standard turbulent velocity and magnetic fields ( $\boldsymbol{U}_{T}$ is the velocity not including the background shear). Throughout this work we consider initially homogenous turbulence with zero average helicity.

\subsection{Non-helical dynamo mechanisms}

To examine field generation mechanisms in this geometry, it is helpful to start by defining mean and fluctuating fields through the relation $\boldsymbol{B}_{T}=\overline{\boldsymbol{B}}_{T}+\boldsymbol{b}=\boldsymbol{B}+\boldsymbol{b}$. Here - is the mean-field average, which is taken to be a spatial average over $x$ and $y$. An average of the induction equation $(2.1 b)$ leads to the well-known mean-field dynamo equations for the mean magnetic field $\boldsymbol{B}$ (Moffatt 1978; Krause \& Rädler 1980),

$$
\partial_{t} \boldsymbol{B}=\nabla \times\left(\boldsymbol{U}_{0} \times \boldsymbol{B}\right)+\nabla \times \mathcal{E}+\frac{1}{R m} \nabla^{2} \boldsymbol{B} .
$$

Here $\mathcal{E}=\overline{\boldsymbol{u} \times \boldsymbol{b}}$ is the EMF, which provides the connection between the small-scale turbulence and large-scale fields. If we assume scale separation between the mean and fluctuating fields, a Taylor expansion of $\mathcal{E}$ leads to the form

$$
\mathcal{E}_{i}=\alpha_{i j} B_{j}-\eta_{i j} J_{j}+\cdots,
$$


where $\alpha_{i j}$ and $\eta_{i j}$ are the transport coefficients, and the lack of $(x, y)$ dependence of the mean fields has been used to reduce the number of $\eta$ coefficients from 27 to 4 (note that $B_{z}=0$ ). In the case where the mean fields can be considered a small perturbation to some background turbulent state specified by statistics of $\boldsymbol{u}$ and $\boldsymbol{b}$ (which are influenced by shear and rotation), $\alpha$ and $\eta$ must be independent of $\boldsymbol{B}$.

Due to reflectional symmetry, with a non-helical forcing function $\sigma_{u}$, the $\alpha_{i j}$ coefficients are constrained to vanish on average in this geometry. Instead, we shall study the possibility of a mean-field dynamo that arises purely from the off-diagonal components of $\eta_{i j}$, which can be non-zero due to the anisotropy of the turbulence. Combining (2.2) and (2.3), one obtains

$$
\begin{gathered}
\partial_{t} B_{x}=-\alpha_{y x} \partial_{z} B_{x}-\alpha_{y y} \partial_{z} B_{y}-\eta_{y x} \partial_{z}^{2} B_{y}+\left(\eta_{y y}+\bar{\eta}\right) \partial_{z}^{2} B_{x}, \\
\partial_{t} B_{y}=-S B_{x}+\alpha_{x x} \partial_{z} B_{x}+\alpha_{x y} \partial_{z} B_{y}-\eta_{x y} \partial_{z}^{2} B_{x}+\left(\eta_{x x}+\bar{\eta}\right) \partial_{z}^{2} B_{y},
\end{gathered}
$$

where the time average of the $\alpha_{i j}$ components must vanish. From these equations (with $\alpha_{i j}=0$ ), it is straightforward to show that an eigenmode with the spatial structure $B_{i}=B_{i 0} \mathrm{e}^{\mathrm{i} k z}$ has the growth rate

$$
\gamma_{\eta}=k \sqrt{\eta_{y x}\left(-S+k^{2} \eta_{x y}\right)}-k^{2} \eta_{t}
$$

where we have set $\eta_{y y}=\eta_{x x}=\eta_{t}$ for simplicity. Neglecting $\eta_{x y}$ by assuming $\left|k^{2} \eta_{x y}\right| \ll S$ (for all $k$ for which scale separation holds), one finds that positive dynamo growth is possible if $-S \eta_{y x}>0$ and $k \sqrt{-\eta_{y x} S}>k^{2} \eta_{t}$. The physical mechanism for the instability involves the $B_{x}$ generated by $B_{y}$ (through $-\eta_{y x} \partial_{z}^{2} B_{y}$ ) feeding back on $B_{y}$ through stretching by the mean shear flow (the $-S B_{x}$ term in (2.4)). Thus the possibility of such a non-helical dynamo rests crucially on the phase between $B_{x}$ and $B_{y}$ and therefore on the transport coefficient $\eta_{y x}$, which must be less than zero.

Whether $\eta_{y x}$ is positive or negative depends on the properties of the turbulence in question, in particular, on the sign of $(\overline{\boldsymbol{u} \times \boldsymbol{b}})_{y}$ that arises in the presence of a $B_{y}$ gradient. The standard kinematic approach in dynamo theory has been to consider strong underlying hydrodynamic fluctuations (denoted by $\boldsymbol{u}_{0}$ ), which generate $\boldsymbol{b}$ fluctuations through interaction with $\nabla \boldsymbol{B}$ (and $\boldsymbol{B}$ ). Although various early analytic works argued for a kinematic shear-current dynamo of this type (Urpin 1999, 2002; Rogachevskii \& Kleeorin 2003), subsequently several authors found that kinematically $\eta_{y x}>0$ (at least at low $\mathrm{Rm}$ ) and thus concluded that a coherent kinematic dynamo cannot explain the field generation observed in numerical experiments (Rädler \& Stepanov 2006, Brandenburg et al. 2008a, Singh \& Jingade 2015; Paper II). Here we argue instead that strong homogenous magnetic fluctuations (denoted by $\boldsymbol{b}_{0}$ ) can generate $\boldsymbol{u}$ fluctuations with the required correlations to cause a negative $\eta_{y x}$. Such $\boldsymbol{b}_{0}$ fluctuations should be ubiquitous in MHD turbulence at high Reynolds numbers, since the small-scale dynamo will be unstable (with a large growth rate set by the smallest scales in the turbulence), creating a turbulent state with $\boldsymbol{b}_{0} \sim \boldsymbol{u}_{0}$ (Schekochihin et al. 2004).

Before continuing, it is worth mentioning another possibility for large-scale field generation in this geometry - the so-called, stochastic- $\alpha$ effect. This arises through fluctuations in the $\alpha_{i j}$ coefficients, even though their mean must vanish (Vishniac \& Brandenburg 1997; Heinemann, McWilliams \& Schekochihin 2011; Mitra \& Brandenburg 2012). This dynamo is not mean field in the usual sense since it relies on the finite size of the system to cause the $\alpha$ fluctuations that lead to mean-field 
growth; nonetheless, given that the universe is sampling a single realization of turbulence, not the ensemble average, such effects could be entirely physical. (That said, one consequence of this incoherent dynamo mechanism is that the growth rate can be arbitrarily increased or decreased by changing the volume of the mean-field average, which hints that coherent effects should dominate when a very large range of scales are present.) While we shall not examine the stochastic- $\alpha$ effect in detail in this work (see Paper II), it is important to be mindful of the possibility, since it complicates the analysis of simulation results where large-scale field growth is observed. One distinguishing feature from the shear-current effect is that $\boldsymbol{B}(z, t)$ cannot have a constant phase in time as it grows, since the average of $\boldsymbol{B}$ over an ensemble of realizations vanishes, implying $\boldsymbol{B}$ must be uncorrelated with itself after $t \gtrsim\left(k^{2} \eta_{t}\right)^{-1 \dagger}$. More information, including analyses of the relative importance of the coherent and incoherent shear-dynamo mechanisms in low- $R m$ systems, can be found in Paper II.

\subsection{The mechanism for the magnetic shear-current effect}

In this section we discuss the mean-field generation mechanism of the magnetic shearcurrent effect. The stability analysis given in $\S 2.1$ makes it clear that we require $\eta_{y x}<0$ for a coherent dynamo instability. In the present context, with both the mean magnetic field and flow in the $y$ direction and their prescribed spatial dependencies (see figure $3 a$ ), this is equivalent to requiring that the $y$ component of the turbulent EMF be negative. The challenge is then for us to explain how this can come about in the present geometry. The cartoon picture that we present has its origins in the analytic 'second-order correlation approximation' (SOCA) calculations presented in Paper III. In particular, by selectively removing terms from the calculation and examining the effects on the final $\eta_{y x}$, one can unambiguously determine from where the effect arises (at least within the quasi-linear approximation). Most importantly, this exercise shows that the magnetic shear-current effect arises exclusively from the pressure response of the velocity fluctuations. The mechanism is fundamentally related to the lack of turbulent resistivity quenching by the magnetic field (often referred to as a lack of ' $\beta$ quenching'; see Gruzinov \& Diamond (1994) and Bhattacharjee \& Yuan (1995)), which results from a cancellation between a turbulent magnetic resistivity (of the same form as kinematic turbulent resistivity), and an equal and opposite contribution from the pressure response (Avinash 1991).

We divide our discussion up as answers to three questions: (i) how do we generate the fluctuations needed to support the required EMF? (ii) What happens in the absence of flow shear? And (iii) what happens in the presence of flow shear?

\subsubsection{How do we generate the fluctuations needed to support the required EMF?}

The fluctuations needed to support our physical picture are magnetically driven. In contrast to kinematic dynamos, the Maxwell stress $\boldsymbol{B}_{T} \cdot \boldsymbol{\nabla} \boldsymbol{B}_{T}$ is fundamental for a magnetically driven dynamo, since this is required to generate $\boldsymbol{u}$ from $\boldsymbol{b}$ (in the same way the Lorentz force $\boldsymbol{\nabla} \times\left(\boldsymbol{U}_{T} \times \boldsymbol{B}_{T}\right)$ generates correlated $\boldsymbol{b}$ fluctuations in kinematic dynamos). Such dynamos can still be analysed linearly if one assumes that

\footnotetext{
$\dagger$ This condition may be altered if one considers the effects of magnetic helicity conservation, which may cause a local magnetic $\alpha$ effect as the large-scale field grows (Brandenburg et al. 2008a). However, this also causes coupling between different mean-field modes, and the detailed consequences of such an effect remain unclear.
} 


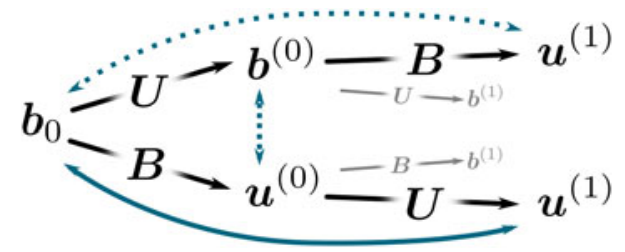

FIGURE 1. Depiction of the interactions between fluctuations $\left(\boldsymbol{b}_{0}, \boldsymbol{u}^{(i)}\right.$ and $\left.\boldsymbol{b}^{(i)}\right)$ and a mean magnetic field $\boldsymbol{B}$ or a shear flow $\boldsymbol{U}$, that can lead to a non-zero shear-current effect through $\overline{\boldsymbol{u} \times \boldsymbol{b}}$, starting from strong homogenous magnetic fluctuations $\boldsymbol{b}_{0}$. Here the straight black arrows, with either $\boldsymbol{B}$ or $\boldsymbol{U}$, depict an interaction that creates one fluctuating field from another, which will be correlated with the original fluctuation and thus can contribute to the EMF (for instance $\boldsymbol{u}^{(0)} \sim \tau_{c} \boldsymbol{B} \cdot \nabla \boldsymbol{b}_{0}+\tau_{c} \boldsymbol{b}_{0} \cdot \nabla \boldsymbol{B}$ ). The double headed (blue) arrows indicate the lowest-order combinations of $\boldsymbol{b}_{0}, \boldsymbol{u}^{(i)}$ and $\boldsymbol{b}^{(i)}$ that can lead to non-zero $\eta_{y x}$, with the interaction studied in $\$ 2.2$ shown by the solid line.

the interaction of fluctuations with mean fields is more important for the EMF than the interaction with themselves; that is,

$$
\boldsymbol{b} \cdot \nabla \boldsymbol{B}+\boldsymbol{B} \cdot \nabla \boldsymbol{b} \text { is more important than } \boldsymbol{b} \cdot \nabla \boldsymbol{b}-\overline{\boldsymbol{b} \cdot \nabla \boldsymbol{b}} .
$$

This approximation - which along with a similar approximation for the Lorentz force, is the basis for SOCA - is valid only at low Reynolds numbers and non-zero mean fields, but allows one to consider how small-scale eddies and field loops would interact with large-scale field and flow gradients in a relatively straightforward way. Note that, 'is more important' in (2.6) refers to the terms' relative importance for the generation of an EMF that is correlated with $\boldsymbol{B}$ (this correlation is necessary for a large-scale dynamo). Since only the part of $\boldsymbol{b}$ that is influenced by $\boldsymbol{B}$ can contribute to this correlation, it seems reasonable to surmise that results should be qualitatively applicable outside their true validity range. In other words, since the interaction of $\boldsymbol{b}$ with $\boldsymbol{B}$ is the cause of the magnetic shear-current effect in the first place, we shall focus on this (rather than the much more complicated nonlinear terms) for the development of our simple cartoon model.

The shear-current effect requires both a field gradient and a flow gradient (shear flow). Thus, any perturbation $\boldsymbol{b}_{0}$ (arising as part of the bath of statistically homogenous magnetic fluctuations) must interact with both $\boldsymbol{U}$ and $\boldsymbol{B}$ to generate a $\boldsymbol{u}$ fluctuation. The possible ways in which this can happen are illustrated in figure 1, where the notation is the same as that used in Paper III, with $\boldsymbol{f}^{(0)}$ indicating a field that arises directly from the interaction of $\boldsymbol{b}_{0}\left(\right.$ or $\left.\boldsymbol{u}_{0}\right)$ with the mean fields, and $\boldsymbol{f}^{(1)}$ indicating one that arises through $\boldsymbol{f}^{(0)}$. In addition, we use $(\cdot)_{b}$ to denote the part of a transport coefficient that is due to homogenous magnetic fluctuations; for example, $\left(\eta_{y x}\right)_{b}$. From the momentum equation, a $\boldsymbol{b}$ perturbation can generate a $\boldsymbol{u}$ perturbation through $\boldsymbol{b} \cdot \boldsymbol{\nabla} \boldsymbol{B}+\boldsymbol{B} \cdot \boldsymbol{\nabla} \boldsymbol{b}$, while a $\boldsymbol{u}$ perturbation can generate a $\boldsymbol{u}$ perturbation through $-\boldsymbol{u} \cdot \nabla \boldsymbol{U}-\boldsymbol{U} \cdot \nabla \boldsymbol{u}$. Similarly, from the induction equation a $\boldsymbol{b}$ perturbation is generated through either a $\boldsymbol{u}$ perturbation $(\boldsymbol{B} \cdot \nabla \boldsymbol{u})$, or through a $\boldsymbol{b}$ perturbation $(-\boldsymbol{U} \cdot \nabla \boldsymbol{b})$. We see from figure 1 that there are three possibilities for contributing to $\left(\eta_{y x}\right)_{b}: \boldsymbol{u}^{(0)} \times \boldsymbol{b}^{(0)},\left(\boldsymbol{u}^{(1)} \times \boldsymbol{b}_{0}\right)_{1}$ and $\left(\boldsymbol{u}^{(1)} \times \boldsymbol{b}_{0}\right)_{2}$. Here $\left(\boldsymbol{u}^{(1)} \times \boldsymbol{b}_{0}\right)_{1}$ refers to the pathway for generating $\boldsymbol{u}^{(1)}$ through $\boldsymbol{u}^{(0)}$ (shown by the solid arrow in figure 1), while $\left(\boldsymbol{u}^{(1)} \times \boldsymbol{b}_{0}\right)_{2}$ refers to the pathway through $\boldsymbol{b}^{(0)}$ (shown by the top dashed arrow). Out of these, we have determined from the calculations in Paper III that $\left(\boldsymbol{u}^{(1)} \times \boldsymbol{b}_{0}\right)_{1}$ is 
both the simplest and contributes the most to $\left(\eta_{y x}\right)_{b}$. In particular, the mechanism does not directly rely on dissipation to generate the required correlations, as will be seen below ${ }^{\ddagger}$. We have found empirically that the $\boldsymbol{u}^{(0)} \times \boldsymbol{b}^{(0)}$ contribution is moderate in size (generally a factor of $\sim 2$ smaller than $\boldsymbol{u}^{(1)} \times \boldsymbol{b}_{0}$ ) and also always negative, while the $\left(\boldsymbol{u}^{(1)} \times \boldsymbol{b}_{0}\right)_{2}$ contribution (dotted line in figure 1) can change sign but is much smaller in magnitude.

\subsubsection{What happens in the absence of flow shear?}

As mentioned above, in the absence of flow shear, there is no quenching of the turbulent resistivity. This effect - which could also be stated as $\left(\eta_{x x}\right)_{b}=\left(\eta_{y y}\right)_{b}=0$ in the notation of (2.4) - arises through the pressure response of the fluid. We feel it helpful to first explain this mechanism in more detail, since the form of the pressure response has not been discussed in detail in previous literature (so far as we are aware $)^{\S}$ and the magnetic shear-current effect is essentially an extension of this. As can be seen using SOCA (or the $\tau$ approximation; see Rädler, Kleeorin \& Rogachevskii 2003), the effect occurs because the pressure response has an equal and opposite effect to the primary velocity perturbation (Avinash 1991). This behaviour is illustrated graphically in figure 2, which shows the response of the fluid to a magnetic perturbation in the linearly varying magnetic field $\boldsymbol{B}=S_{B} z \hat{\boldsymbol{y}}$. Due to the mean-field geometry, the velocity perturbation $\delta \boldsymbol{u}_{\text {basic }}^{(0)} \sim \tau_{c} \boldsymbol{b} \cdot \nabla \boldsymbol{B}$ (where $\tau_{c}$ is some turbulent correlation time) is simply $S_{B} b_{0} \hat{\boldsymbol{y}}$; i.e. only the $z$ component of $\boldsymbol{b}_{0}$ contributes. Note that the other contribution $\delta \boldsymbol{u}_{\text {basic }}^{(0)} \sim \tau_{c} \boldsymbol{B} \cdot \nabla \boldsymbol{b}$, will only contribute directly to the EMF if there is a mean correlation between $\boldsymbol{b}$ and $\boldsymbol{\nabla} \boldsymbol{b}$, which occurs if there is net current helicity (this term is the origin of the magnetic $\alpha$ effect) ${ }^{\mathrm{T}}$. Obviously, the perturbation $\delta \boldsymbol{u}_{\text {basic }}^{(0)} \sim S_{B} b_{0} \hat{\boldsymbol{y}}$ is correlated with $\boldsymbol{b}_{0}$ and it is straightforward to see (see figure $2 b$ ) that a net $\mathcal{E}$ is created in the $\hat{\boldsymbol{x}}$ direction, opposite to the mean current and thus acting as a turbulent dissipation for the mean field.

However, as is clear from figure 2, the $\delta \boldsymbol{u}_{\text {basic }}^{(0)}$ perturbation is not divergence free, given any $y$ variation in $b_{0 z}$. In figure $2(c)$, the shaded regions illustrate where the divergence of $\delta \boldsymbol{u}_{\text {basic }}^{(0)}$ is positive (yellow) or negative (red). Given the incompressibility of the fluid, a non-zero divergence is not possible, and the $\nabla p$ term responds appropriately, creating a flow perturbation from regions of negative divergence to positive divergence (mathematically, $\delta \boldsymbol{u}_{\text {pres }}^{(0)}=\nabla^{-2}\left[-\nabla\left(\nabla \cdot \delta \boldsymbol{u}_{\text {basic }}^{(0)}\right)\right]$ ). As shown in figure $2(c)$ this perturbation is anticorrelated with $\delta \boldsymbol{u}_{\text {basic }}^{(0)}$ and thus creates an oppositely directed EMF, in the $+\boldsymbol{J}$ direction. Further, since $\nabla \cdot(\boldsymbol{b} \cdot \nabla \boldsymbol{B})=\boldsymbol{\nabla} \cdot(\boldsymbol{B} \cdot \nabla \boldsymbol{b})$, each of these linear contributions to the Maxwell stress add in the same way to the pressure perturbation, and a more careful calculation shows that the effect exactly cancels the original EMF on average. Given its reliance on the pressure response, the effect

\footnotetext{
${ }^{\ddagger}$ To be more specific, in the SOCA calculations, this contribution does not involve $k$ derivatives of the functions $E_{\eta}=1 /\left(\mathrm{i} \omega-\bar{\eta} k^{2}\right)$ or $N_{v}=1 /\left(\mathrm{i} \omega-\bar{v} k^{2}\right)$ (which are zero at $\bar{v}=0$ or $\bar{\eta}=0$ ), while the other two contributions do.

${ }^{\S}$ Yokoi (2013) gives a slightly different model for the negative contribution to the magnetic resistivity, based on small-scale current perturbations creating a magnetic pressure. This model is similar to that presented here but does not directly include the fluid pressure (it includes the magnetic pressure), which we have seen to be important by studying the relevant terms in SOCA calculations.

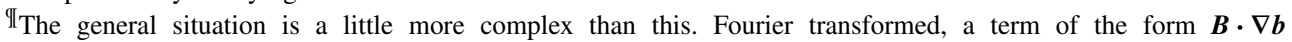
becomes $\mathrm{i} \boldsymbol{B} \cdot \boldsymbol{k} \boldsymbol{b}-(\boldsymbol{\nabla} \boldsymbol{B})_{j l} k_{j} \partial_{k_{l}} b_{i}+\cdots$ (where the Einstein summation convention is used). Without helicity the first term does not contribute when averaged over a domain, but the second term can in general be non-zero. However, contributions of this form generally seem to be smaller in magnitude, and its dependence on the $\boldsymbol{k}$ derivative of the fluctuations makes it troublesome to arrive at a simple cartoon picture. See Paper III for more detail on the mathematics of the calculation.
} 


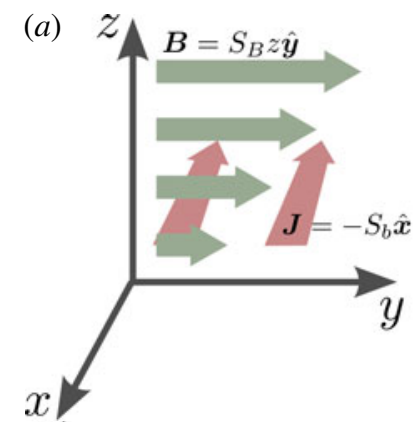

(b)

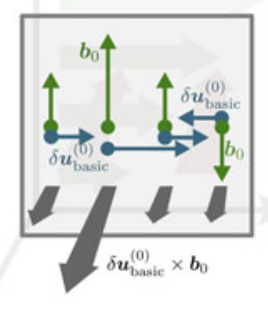

(c)

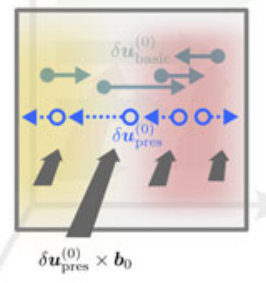

FIGURE 2. Graphical illustration of the mean-field resistivity - or lack thereof - generated by homogenous small-scale magnetic fluctuations, with the geometry of the mean field illustrated in $(a)$. (b) Shows how $b_{0 z}$ perturbations (from an homogeneous turbulent bath) lead to a $\boldsymbol{u}$ perturbation (labelled $\delta \boldsymbol{u}_{\text {basic }}^{(0)}$ ) through $\boldsymbol{b} \cdot \nabla \boldsymbol{B}=S_{B} b_{0 z} \hat{\boldsymbol{y}}$, resulting in an $\mathrm{EMF}$ in the $-\boldsymbol{J}$ direction. (c) Shows how the pressure response to this $\delta \boldsymbol{u}_{\text {basic }}^{(0)}$ (labelled $\delta \boldsymbol{u}_{\text {pres }}^{(0)}$ ), which arises due to its non-zero divergence (yellow and red shaded regions for $\boldsymbol{\nabla} \cdot \delta \boldsymbol{u}_{\text {basic }}^{(0)}>0$ and $\boldsymbol{\nabla} \cdot \delta \boldsymbol{u}_{\text {basic }}^{(0)}<0$ respectively), leads to an EMF that opposes that from $\delta \boldsymbol{u}_{\text {basic }}^{(0)}$. A more careful calculation shows that the cancellation is exact (in incompressible turbulence at low $R m$ ), so the turbulent resistivity due to magnetic fluctuations vanishes. See text for further discussion.

will be reduced in a compressible flow (presumably becoming negligible for high Mach number flows), and one would expect $\boldsymbol{b}_{0}$ fluctuations to increase the turbulent diffusivity in this case (the magnetic shear-current effect will also be less effective in a compressible flow). Finally, it is worth mentioning that $z$ variation of the initial $\boldsymbol{b}_{0}$ perturbation will not contribute since this creates a $\left(\delta \boldsymbol{u}_{\text {pres }}^{(0)}\right)_{z}$ (which is zero in a cross-product with $\left.b_{0 z}\right)$, while $x$ variation of $\boldsymbol{b}_{0}$ produces a $\left(\delta \boldsymbol{u}_{\text {pres }}^{(0)}\right)_{x}$ that is out of phase with the original $b_{0 z}$ perturbation.

\subsubsection{What happens in the presence of flow shear?}

In the presence of flow shear, the cancellation discussed in the previous section leaves a residual $x$-directed $\boldsymbol{u}$ perturbation. This perturbation - which arises from the interaction of the pressure perturbation in figure 2 with the mean shear, followed by the pressure response to this secondary perturbation - leads to the magnetic shearcurrent effect. This rather complex process is illustrated in graphically in figure 3, using similar conventions (and colour schemes) to figure 2. A shear flow in the $\hat{\boldsymbol{y}}$ direction is included in addition to the mean field $\boldsymbol{B}=S_{B} z \hat{\boldsymbol{y}}$, which corresponds exactly to the geometry discussed in $\S 2.1$ and $(2.4 b)$. Recall that $\eta_{y x}<0$ is equivalent to $\mathcal{E}_{y}<0$ in this geometry (see (3.2)). Figure 3(b) illustrates the same effect as shown in figure 2, now including $x$ and $y$ dependence of the $b_{0 z}$ perturbation. As is evident, even though $\delta \boldsymbol{u}_{\text {basic }}^{(0)}$ points only in the $y$ direction, the pressure response includes equally strong $x$ directed flows, since it arises from the spatial dependence of $\boldsymbol{\nabla} \cdot \delta \boldsymbol{u}_{\text {basic }}^{(0)}$. The resulting $\left(\delta \boldsymbol{u}^{(0)}\right)_{x}$ is out of phase with $\boldsymbol{b}_{0}$, so does not contribute to an EMF itself, but it is sheared by the background flow through $\delta \boldsymbol{u}_{\text {basic }}^{(1)} \sim-\tau_{c} \boldsymbol{u}^{(0)} \cdot \nabla \boldsymbol{U}=\tau_{x} S u_{x}^{(0)} \hat{\boldsymbol{y}}$, which is shown in figure $3(b)$. Again, since only the $x$ component contributes, $\delta \boldsymbol{u}_{\text {basic }}^{(1)}$ is not divergence free (shaded yellow and red regions for $\boldsymbol{\nabla} \cdot \delta \boldsymbol{u}_{\text {basic }}^{(1)}>0$ and $\boldsymbol{\nabla}$. $\delta \boldsymbol{u}_{\text {basic }}^{(1)}<0$ respectively). We see that the $x$ component of the pressure response towards (away from) regions where $\nabla \cdot \delta \boldsymbol{u}_{\text {basic }}^{(1)}>0\left(\nabla \cdot \delta \boldsymbol{u}_{\text {basic }}^{(1)}<0\right)$ is now correlated and 

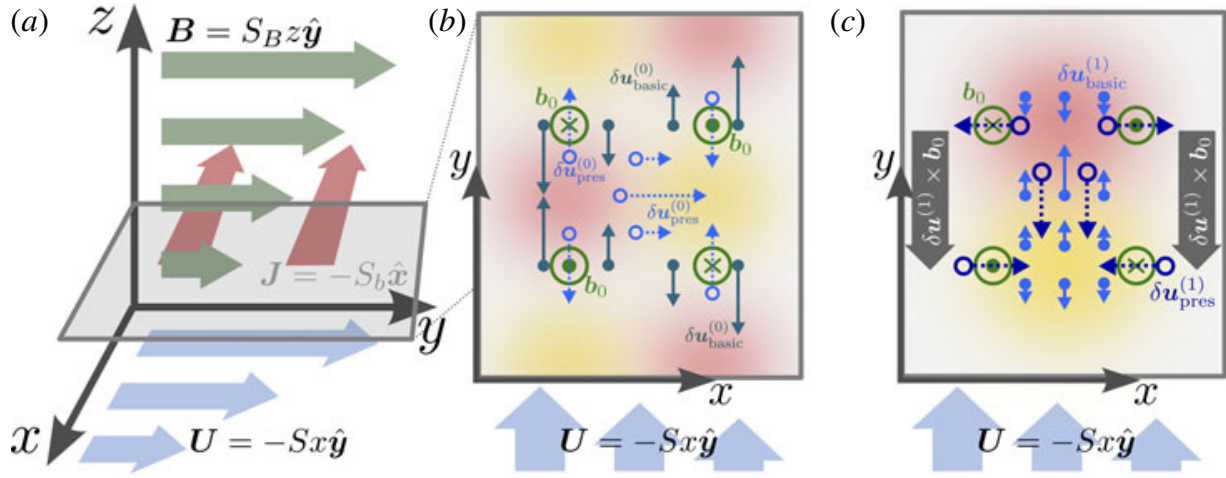

FIGURE 3. Graphical illustration of the magnetic shear-current effect, which should be interpreted as follows. (a) The geometry of the mean field and shear flow. (b) The flow perturbation (both $\delta \boldsymbol{u}_{\text {basic }}^{(0)}$ and $\delta \boldsymbol{u}_{\text {pres }}^{(0)}$ ) that arises due to $x, y$ dependence of the initial $b_{0 z}$, before interaction with the shear flow (note the rotation of the axes compared to $(a)$ ). (c) The $\delta \boldsymbol{u}^{(1)}$ perturbation that arises from $\delta \boldsymbol{u}^{(0)}$ due to stretching by the flow, which illustrates a correlation between $\delta \boldsymbol{u}_{\text {pres }}^{(1)}$ and the original $b_{0 z}$ structure. The resulting $\mathcal{E}$ is pointing in the $-\hat{\boldsymbol{y}}$ direction, corresponding to a negative $\eta_{y x}$. (b) The yellow (red) shading indicates where $\delta \boldsymbol{u}_{\text {basic }}^{(0)}$ has a positive (negative) divergence, while the shading in panel (c) shows the same for $\delta \boldsymbol{u}_{\text {basic }}^{(1)}$. More information and discussion is given in the main text.

in phase with the original perturbation. Most importantly, its direction is such that $\mathcal{E}=\delta \boldsymbol{u}^{(1)} \times \boldsymbol{b}_{0}$ is always in the $-\hat{\boldsymbol{y}}$ direction, leading to $\left(\eta_{y x}\right)_{b}<0$. Note that here, unlike in discussion of figure 2, the effect relies on the $x$ component of the pressure response (perpendicular to $\delta \boldsymbol{u}_{\text {basic }}$ ), which must occur for any perturbation that varies in $x$ because the response is the gradient of a scalar field (i.e. $-\nabla p$ ).

At this point, the reader could be forgiven for viewing the magnetic shear-current mechanism explained above with some scepticism - how do we know there are no opposing mechanisms to cancel out such effects? The simplest answer is that we have derived the physical picture in figure 3 from the SOCA calculation, by noting that $\left(\eta_{y x}\right)_{b}$ is unchanged by removal of all contributions to the velocity perturbation other than $\nabla p$, and through the exploration of the different pathways in figure 1. More physically, the reason the pressure is necessary for the shear-current effect arises from the mean field and flow geometry. In particular, if a small-scale fluctuation interacts with either $\boldsymbol{U}$ or $\boldsymbol{B}$ through $\boldsymbol{u} \cdot \nabla \boldsymbol{U}, \boldsymbol{u} \cdot \nabla \boldsymbol{B}, \boldsymbol{b} \cdot \nabla \boldsymbol{U}$ or $\boldsymbol{b} \cdot \nabla \boldsymbol{B}$, the resulting perturbation is always in the $\pm \hat{\boldsymbol{y}}$ direction. Obviously, such a perturbation cannot lead to a non-zero $\mathcal{E}_{y}$. Thus, $\eta_{y x}$ is both very important for dynamo action and particularly complicated to generate, because the flow and mean field are in the same direction as the required EMF. This explains why $\eta_{y x}$ is seen to be much smaller than $\eta_{x y}$ in numerical simulation and calculations (Brandenburg et al. 2008a; Singh \& Sridhar 2011; Paper II), as well as the capricious nature of the kinematic shear-current effect (the sign of $\left(\eta_{y x}\right)_{u}$ may depend on the Reynolds numbers, while analytic results depend on the closure method used), for which these same arguments apply". Note that the

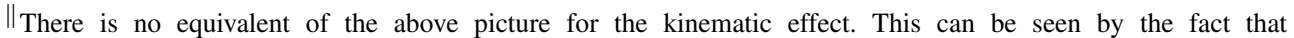
the $\boldsymbol{b}$ perturbation that arises from $\boldsymbol{b} \cdot \boldsymbol{\nabla} \boldsymbol{U}$ is necessarily in the $y$ direction (since there is no pressure). This means the $\boldsymbol{U} \cdot \boldsymbol{\nabla} \boldsymbol{b}$ term, which involves the derivative of $\boldsymbol{b}$ in $\boldsymbol{k}$ space, must be responsible for any $\mathcal{E}_{y}$ (another possibility is correlations between components of $\boldsymbol{u}_{0}$ arising from $\boldsymbol{\nabla} \cdot \boldsymbol{u}_{0}=0$ ). The same is true of the other pathways in figure 1 .
} 
requirements for $\mathcal{E}_{y} \neq 0$ in figure 3 are very specific - a $y$ variation of the $x$ variation of $b_{0 z}$ - and it is straightforward to see that this is the only possibility for generation of a $\delta u_{x}$ in this way. This implies we can ignore both the other $\boldsymbol{b}$ components and any variation in $z$. Thus, although figures 2 and 3 show the fluid response to a rather specific form for $\boldsymbol{b}_{0}$, the important features of the resulting perturbations (shown in panel (c) in each case) are relatively generic for more general $\boldsymbol{b}_{0}$. Further, since the effect is linear, Fourier modes (such as that shown in figure 3) can be added to form a more general $\boldsymbol{b}$ perturbation, and we are sure to obtain $\mathcal{E}_{y}<0$.

We have thus seen how magnetic fluctuations can produce a negative $\eta_{y x}$ through their interaction with large-scale field and flow gradients, which can in some cases lead to large-scale dynamo action. In addition, the reliance of the effect on the fluid pressure response, as well as the general difficulty of creating perturbations that create an EMF parallel to both the mean flow and field, help explain the relative dominance of the magnetic over the kinematic shear-current effect.

\section{Numerical evidence}

In this section we illustrate numerically that the magnetic shear-current mechanism discussed above is indeed realizable in MHD turbulence. We show using direct numerical simulation that it is possible and realizable to have the small-scale dynamo drive the growth of the large-scale dynamo. So far as we are aware, this is the first demonstration of this interesting behaviour.

The methods used to illustrate this effect in numerical simulation are somewhat non-standard in the dynamo literature. In particular, at each set of physical parameters we carry out an ensemble of simulations, each with different noise realizations. We then measure transport coefficients before and after small-scale saturation in each simulation, which shows (after an ensemble average) that $\eta_{y x}$ becomes more negative after the saturation of the small-scale dynamo. That this can drive a coherent dynamo is illustrated by qualitative observation of the mean-field pattern, as well as solution of the mean-field equations (2.4) using the measured transport coefficients. The ensemble of simulations is required as a result of the relatively short period of large-scale dynamo growth before nonlinear saturation effects become significant. This is because the large-scale magnetic field starts its growth (when the small-scale dynamo saturates) at relatively large amplitudes, being in approximate equipartition with the small-scale fluctuations due to the finite size of the mean-field average. We shall see that in many cases, the growth of the mean field lasts little more than $20 \rightarrow 30$ shearing times before saturating, and that its behaviour can vary substantially between realizations. Because of this, the ensemble average over simulations is highly advantageous for accurate determination of the transport coefficients. (In other work we have used statistical simulation to circumvent this problem, but this requires a quasi-linear approximation, which eliminates in small-scale dynamo; see Paper II).

The method for measuring the transport coefficients from simulation data after small-scale dynamo saturation (termed the 'projection method') is also non-standard, and will be explained in some detail. Because test-field methods that explicitly include the magnetic fluctuations are rather complex and in the early stages of development (Rheinhardt \& Brandenburg 2010), we instead choose to measure transport coefficients directly from mean field and EMF data taken from simulations. The method, which is a modified version of that proposed in Brandenburg \& Sokoloff (2002) and is also used with some success in Racine et al. (2011) and Simard, Charbonneau \& Bouchat (2013), involves approximately solving $\mathcal{E}_{i}=\alpha_{i j} B_{j}-\eta_{i j} J_{j}$ at each time step and taking 
spatio-temporal averages to obtain transport coefficients. To ensure that correct results are obtained, the projection method is checked in two independent ways: first, it is used to compute transport coefficients for low- $R m$ kinematic shear dynamos and compared directly to the test-field method (this is presented in appendix A). Second, we solve the mean-field equations using the measured time-dependent transport coefficients and compare to the mean-field evolution from the simulations. This provides a thorough check that the measured coefficients are correct, without relying on any assumptions about the type of dynamo, or simplifications to the form of $\mathcal{E}$.

Calculations are carried out using the nonlinear MHD equations (2.1), with homogenous Cartesian geometry, periodic boundary conditions in the azimuthal $(y)$ and vertical $(z)$ directions and shearing periodic boundary conditions in the radial (x) direction. We use the SNOOPY code (Lesur \& Longaretti 2007), which applies the Fourier pseudospectral method (in the shearing frame), and system rotation is included in some simulations through a mean Coriolis force. The flow field forcing $\left(\sigma_{u}\right.$ in (2.1)) is non-helical, white noise in time, isotropic and centred in wavenumber space at $|\boldsymbol{k}|=6 \pi$ (with width $6 \pi / 5$ ). All simulations presented here use a box of size $\left(L_{x}, L_{y}, L_{z}\right)=(1,4,2)$ with a resolution of $\left(N_{x}, N_{y}, N_{z}\right)=(64,128,128)$, and we take $\bar{\eta}=1 / 2000(R m=2000) P m=8(R e=250)$. To test convergence, we have run several cases (both with and without rotation) at twice the resolution, and there is no discernible difference with lower resolution runs in either the spectrum, turbulence level or mean-field evolution**.

Our choice of this numerical set-up for the simulation ensembles is motivated both by the calculations of Yousef et al. (2008a) with unstable small-scale dynamo (see their figure 9), and from studies of magnetorotational instability (MRI) driven turbulence in the shearing box ${ }^{\dagger \dagger}$. In particular, the relatively low Reynolds numbers are chosen both for computational reasons (100 simulations are run for each parameter set), and so that there is no transition to self-sustaining turbulence if $\sigma_{u}=0$. Thus, we choose Reynolds numbers that are intermediate between the small-scale dynamo being stable (on the low side) and the system transitioning to turbulence in the absence of noise (on the high side). While similar mechanisms may be operating in the case of self-sustaining turbulence (Lesur \& Ogilvie 2008a,b), it is certainly a complicating influence that is more easily ignored for the purposes of this study. The relatively high $P m$ is chosen for the obvious reason of enhancing $\boldsymbol{b}$ in comparison to $\boldsymbol{u}$, while still allowing for a moderate range of scales in $\boldsymbol{u}$. It seems worth emphasizing that we do not consider these measurements to be firm proof of the magnetic shear-current effect's importance at high $R m$; rather, they serve as a demonstration that it is possible for the small-scale dynamo to significantly change $\eta_{y x}$, and as motivation for further studies at higher Reynolds numbers and with different numerical set-ups.

\subsection{Measurement of the transport coefficients}

In this section we describe the methods - the test-field method (Schrinner et al. 2005), and the projection method (based on Brandenburg \& Sokoloff 2002) - for obtaining the transport coefficients from simulations. Those readers who are primarily

\footnotetext{
** Since there are relatively large differences between realizations (see figure 5), we compare a variety of the lower resolution cases with the higher resolution runs, and note that disparities between different low resolution realizations are as severe as those between the low and high resolution runs. We thus conclude that the differences between the lower and higher resolutions are negligible at these parameters, and use the lower resolution for computational reasons in the ensemble of realizations.

$\dagger^{\dagger}$ The numerical set-up, aside from the noise source, is identical that of zero-net-flux unstratified accretion disk simulations.
} 
interested in results may wish to skip directly to $\S 3.2$. Since the projection method is uncommon in the dynamo literature, its accuracy is verified in appendix A through direct comparison to test-field method calculations for low- $R m$ non-helical shear dynamos over a range of $\eta_{y x}$. While the test-field method gives unambiguous answers for kinematic transport coefficients (before the small-scale dynamo saturation), results can become more difficult to interpret in the presence of magnetic fluctuations (Cattaneo \& Hughes 2009; Hubbard et al. 2009; Rheinhardt \& Brandenburg 2010). In contrast, the projection method does not rely on any assumptions regarding the importance of small-scale magnetic fields, operating purely from the mean-field data from a given simulation. In addition to this method, we have also applied a weighted least squares method, fitting simulation data for a single mode (Kowal, Otmianowska-Mazur \& Hanasz 2005). This has led to almost identical results for the low- $R m$ test cases and the main results given here. However, the least squares method was generally found to be somewhat less reliable and rather delicate, and we do not discuss the details. Another possibility for measuring transport coefficients, which could be explored for non-helical shear dynamos in future work, is given in Tobias \& Cattaneo (2013).

\subsubsection{Test-field method}

The test-field method (Schrinner et al. 2005), which is used for calculating transport coefficients before small-scale dynamo saturation, has become a standard tool in dynamo studies (Brandenburg et al. 2008a), so we discuss this only briefly. The method involves solving for a set of $Q$ 'test fields' $\boldsymbol{b}^{q}$ (where $q=1 \rightarrow Q$ ), in addition to the standard MHD equations. The test fields satisfy the small-scale induction equation,

$$
\partial_{t} \boldsymbol{b}^{q}=\nabla \times\left(\boldsymbol{u} \times \boldsymbol{B}^{q}\right)+\nabla \times\left(\boldsymbol{U} \times \boldsymbol{b}^{q}\right)+\nabla \times\left(\boldsymbol{u} \times \boldsymbol{b}^{q}-\overline{\boldsymbol{u} \times \boldsymbol{b}^{q}}\right)+\bar{\eta} \nabla^{2} \boldsymbol{b}^{q},
$$

where $\boldsymbol{B}^{q}$ are a set of $Q$ test mean fields (specified at the start of the simulation), and $\boldsymbol{u}$ and $\boldsymbol{U}$ are taken from the simulation. By calculating the EMF $\mathcal{E}^{q}=\overline{\boldsymbol{u} \times \boldsymbol{b}^{q}}$ that results from a variety of $\boldsymbol{B}^{q}$, one can determine the transport coefficients. The test-field method's simplest - and most obviously meaningful - use, is to utilize a $\boldsymbol{u}$ field that is unaffected by $\boldsymbol{b}$ or $\boldsymbol{B}$, thus calculating kinematic transport coefficients ${ }^{\ddagger \ddagger}$. A simple extension is the 'quasi-kinematic' method (Brandenburg et al. 2008b; Hubbard et al. 2009), for which one runs an MHD simulation in which $\boldsymbol{u}$ is influenced by self-consistent magnetic fields, and extracts $\boldsymbol{u}$ to insert into the test-field equations. This can most obviously be used to understand how the modification of $\boldsymbol{u}$ by $\boldsymbol{b}$ or $\boldsymbol{B}$ affects the kinematic coefficients (see, for example, Gressel, Bendre \& Elstner 2013), but the direct effect of $\boldsymbol{b}$ fluctuations is not included. A variety of subtleties exist, however, and care must be used in interpreting results; see Hubbard et al. (2009).

\subsubsection{Projection method}

Inclusion of the direct effect of $\boldsymbol{b}$ on transport coefficients in the test-field method introduces significant complications and ambiguities, primarily because it can be difficult to ensure that the test fields $\boldsymbol{b}^{q}$ and $\boldsymbol{u}^{q}$ are linear in the test mean fields. A method has been proposed and explored in Rheinhardt \& Brandenburg (2010);

\footnotetext{
$\ddagger \ddagger$ One complication is the small-scale dynamo of the test fields, which can be unstable and result in exponential growth of $\boldsymbol{b}^{q}$. This can be circumvented by resetting $\boldsymbol{b}^{q}$ periodically (every $T_{\text {reset }}$ ), such that it does not become large in comparison to $\boldsymbol{u}$, but it is important to ensure results are independent of the choice of $T_{\text {reset }}$.
} 
however, given its complications and early stage of development, we choose to use the projection method detailed below to calculate mean-field transport coefficients after small-scale dynamo saturation. This method makes no assumptions regarding the importance of small-scale magnetic fluctuations, simply utilizing mean field and EMF data extracted from standard MHD simulation.

The starting point of the method is the standard Taylor expansion of $\mathcal{E}$ in terms of $\boldsymbol{B}$. In coordinates this is (cf. (2.4)),

$$
\begin{aligned}
& \mathcal{E}_{x}=\alpha_{x x} B_{x}+\alpha_{x y} B_{y}-\eta_{x y} \partial_{z} B_{x}+\eta_{x x} \partial_{z} B_{y}, \\
& \mathcal{E}_{y}=\alpha_{y x} B_{x}+\alpha_{y y} B_{y}-\eta_{y y} \partial_{z} B_{x}+\eta_{y x} \partial_{z} B_{y} .
\end{aligned}
$$

Note that we have not necessarily assumed linearity in $\boldsymbol{B}$, since $\alpha_{i j}$ and $\eta_{i j}$ are not assumed constant. The basic idea of the projection method, proposed in Brandenburg \& Sokoloff (2002), is to extract time-series data for $\mathcal{E}_{i}$ and $B_{i}$ from nonlinear simulation, solving for the transport coefficients in (3.2) at each time point. In principle, all coefficients can be solved for directly, given $\boldsymbol{B}$ and $\mathcal{E}$ data that consists of at least 2 Fourier modes. One calculates

$$
\boldsymbol{E}^{(i)}=\left(\left\langle B_{x} \mathcal{E}_{i}\right\rangle,\left\langle B_{y} \mathcal{E}_{i}\right\rangle,\left\langle\partial_{z} B_{x} \mathcal{E}_{i}\right\rangle,\left\langle\partial_{z} B_{x} \mathcal{E}_{i}\right\rangle\right)^{\mathrm{T}}
$$

and the matrix

$$
\boldsymbol{M}=\left(\begin{array}{cccc}
\left\langle B_{x} B_{x}\right\rangle & \left\langle B_{x} B_{y}\right\rangle & \left\langle B_{x} \partial_{z} B_{x}\right\rangle & \left\langle B_{x} \partial_{z} B_{x}\right\rangle \\
\left\langle B_{y} B_{x}\right\rangle & \left\langle B_{y} B_{y}\right\rangle & \left\langle B_{y} \partial_{z} B_{x}\right\rangle & \left\langle B_{y} \partial_{z} B_{y}\right\rangle \\
\left\langle\partial_{z} B_{x} B_{x}\right\rangle & \left\langle\partial_{z} B_{x} B_{y}\right\rangle & \left\langle\partial_{z} B_{x} \partial_{z} B_{x}\right\rangle & \left\langle\partial_{z} B_{x} \partial_{z} B_{y}\right\rangle \\
\left\langle\partial_{z} B_{y} B_{x}\right\rangle & \left\langle\partial_{z} B_{y} B_{y}\right\rangle & \left\langle\partial_{z} B_{y} \partial_{z} B_{x}\right\rangle & \left\langle\partial_{z} B_{y} \partial_{z} B_{y}\right\rangle
\end{array}\right),
$$

where $\langle\cdot\rangle$ here denotes an average over $z$ and possibly time (the system statistically homogenous in $z$ ). Then, solving

$$
\boldsymbol{E}^{(i)}=\boldsymbol{M} \boldsymbol{C}^{(i)},
$$

for $C^{(1)}=\left(\alpha_{x x}, \alpha_{x y},-\eta_{x y}, \eta_{x x}\right), C^{(2)}=\left(\alpha_{y x}, \alpha_{y y},-\eta_{y y}, \eta_{y x}\right)$, one obtains the full set of transport coefficients.

The data for $\mathcal{E}$ and $\boldsymbol{B}$ are generally quite noisy and some care is required to avoid spurious effects that lead to incorrect results. In particular, while pure white noise in each variable will average to zero over time, there are correlations between components that can significantly pollute the data. These correlations arise from the fact that (3.2) is not the only expected relationship between components of $\boldsymbol{B}$ and $\mathcal{E}$; $\boldsymbol{B}$ is also directly driven by $\mathcal{E}$, and itself, through

$$
\partial_{t} \boldsymbol{B}=-S B_{x} \hat{\boldsymbol{y}}+\nabla \times \mathcal{E}+\bar{\eta} \Delta \boldsymbol{B} .
$$

From (3.6) and by examining data, it is found that the most harmful of the correlations are a correlation between $B_{x}$ and $B_{y}$ (as expected due to $-S B_{x}$ in (3.6)) and a correlation between fluctuations in $\mathcal{E}_{y}$ and $B_{x}\left(B_{x} \text { is directly driven by } \partial_{z} \mathcal{E}_{y}\right)^{\S \S}$. Note that this correlation of $\mathcal{E}_{y}$ and $B_{x}$ is not the same as a non-zero $\alpha_{y x}$ or $\eta_{y y}$ coefficient. Specifically, a noisy change in the imaginary part of $\mathcal{E}_{y}$ by $\epsilon$ will cause a change in

$\S_{\S}$ The correlation between $B_{y}$ and $\mathcal{E}_{x}$ is not so damaging as that between $B_{x}$ and $\mathcal{E}_{y}$ due to the $-S B_{x}$ term in the $B_{y}$ equation and larger range of $B_{y}$ values explored throughout a simulation. 
$B_{x}$ of $\sim k \epsilon \Delta t$ after some time $\Delta t$ (related to the correlation time of the $\mathcal{E}_{\mathrm{y}}$ noise). If the noise fluctuations are of similar or larger magnitude than the range of $B_{x}$ and $\mathcal{E}_{y}$ explored over the course of the calculation, this correlation can cause a negative value for the fit parameter $\eta_{y y}$, since the scatter of the data has a preferred slope. In fact, a consistently negative calculated value for $\eta_{y y}$ is the most prominent spurious effect in simulations, which was also noted in Brandenburg \& Sokoloff (2002) without explanation. That this is purely a consequence of the projection method, and not physical, can be established by comparison to test-field calculations (see appendix A). Importantly, the value of $\eta_{y y}$ is coupled to that of $\alpha_{i y}$ and $\eta_{y x}$. This implies one cannot simply ignore this effect and settle with not knowing $\eta_{y y}$, since the average values of other coefficients will also become polluted.

The basic approach to overcoming these issues described above is to minimize the influence of $B_{x}$ on the calculation, to the extent possible. This is motivated by the fact that $B_{x}$ is very noisy in comparison to $B_{y}$, and is involved in both of the aforementioned damaging correlations. The approach works very well for shear dynamos because $B_{x}$ is much smaller than $B_{y}$ (e.g. in the simulations presented in this work, $B_{x}$ is usually between 25 and 150 times smaller than $B_{y}$ depending on the realization). In addition, those transport coefficients that require $B_{x}$ for their calculation (e.g. $\eta_{x y}$ ) are substantially less interesting, since they do not significantly affect the dynamo growth rate. To enable this reduction in the influence of $B_{x}$, two approximations are made to (3.2). The first and most important is to assume that diagonal transport coefficients are equal, $\eta_{y y}=\eta_{x x}$ and $\alpha_{y y}=\alpha_{x x}$. This is not strictly required by the symmetries of the turbulence with shear (Rädler \& Stepanov 2006; Paper III), but a variety of test-field calculations, including those after saturation of the small-scale dynamo (i.e. quasi-kinematic calculations; see Hubbard et al. 2009; Gressel 2010; Gressel \& Pessah 2015), have shown this to be the case to a high degree of accuracy. The second approximation is to neglect $\eta_{x y}$ and $\alpha_{y x}$. This is justified by the fact that $B_{x} \ll B_{y}$ and $\eta_{x y}<\eta_{x x}$ on average, thus its effect on the mean value of $\eta_{x x}$ should be very small. This approximation is not strictly necessary and similar results can be obtained with $\eta_{x y}$ and $\alpha_{y x}$ included; however, these coefficients fluctuate wildly in time (far more than $\eta_{x x}$ for example) and cause increased fluctuations in the values of the other transport coefficients.

It is useful to briefly consider the proportional error in $\eta_{x x}$ and $\eta_{y x}$ that might arise from these approximations. First, in considering the neglect of $\eta_{x y}$, one starts with the conservative estimate $25 B_{x} \approx B_{y}$. Noting that test-field calculations give $\eta_{x y} \sim 0.25 \eta_{x x}$ for the simulations given in the manuscript (see also Brandenburg et al. 2008a), we see that this approximation should cause less than a $1 \%$ systematic error in $\eta_{x x}$. Second, since we are primarily interested in determining $\eta_{y x}$, let us consider the error in $\eta_{y x}$ that results from an error in $\eta_{y y}$ (caused by either the neglect of $\eta_{x y}$ or the assumption $\eta_{x x}=\eta_{y y}$ ). Noting that $B_{x} \sim-k \sqrt{\eta_{y x} / S} B_{y}$ for a coherent shear dynamo, we can estimate that $\mathrm{i} k \eta_{y x} B_{y} \gtrsim \mathrm{i} k \eta_{y y} B_{x}$ when $k \eta_{y y} \lesssim \sqrt{\left|S \eta_{y x}\right|}$. This inequality is satisfied if the coherent dynamo has a positive growth rate; thus, very approximately, at marginality one would expect the proportional errors in $\eta_{y x}$ and $\eta_{y y}$ to be similar. Combining these two conclusions, one should expect the two approximations to cause very little systematic error in the determination of $\eta_{y x}$, despite the coefficient's small values.

To summarize the previous paragraphs, we shall fit

$$
\begin{gathered}
\mathcal{E}_{x}=\alpha_{y y} B_{x}+\alpha_{x y} B_{y}+\eta_{x x} \partial_{z} B_{y}, \\
\mathcal{E}_{y}=\alpha_{y y} B_{y}-\eta_{x x} \partial_{z} B_{x}+\eta_{y x} \partial_{z} B_{y},
\end{gathered}
$$


to simulation data at each time point. Since there are now fewer coefficients than rows of $\boldsymbol{E}^{(i)}$, the matrix equations are solved in the least squares sense. One final difference from the method as utilized in Brandenburg \& Sokoloff (2002) is a filtering of the data to include only the first two Fourier modes. This is done to improve scale separation, since the small scales of the mean field will be dominated by fluctuations due to the finite size of the horizontal average, and cannot be expected to conform to the ansatz in (3.2)

Finally, we note that $\alpha$ coefficients can be excluded from these calculations altogether, and since their average over long times vanishes, this does not affect the results for $\eta_{i j}$. We have chosen to permit non-zero $\alpha$ in all calculations presented below, both as a consistency check and because over shorter time windows $\alpha$ may not average to exactly zero. Nonetheless, repeating all calculations presented below and in appendix A with $\alpha_{i j}=0$ imposed artificially, one obtains the same results (to within the margin of error). This illustrates that in the neglect of transport coefficients considered above (e.g. $\alpha_{y x}$ ), it is only necessary to consider the errors arising from neglect of $\eta$ coefficients, since those due to neglect of $\alpha$ coefficients average to zero.

\subsubsection{Verification}

To ensure the accuracy of results - especially with regards to possible systematic errors - it is crucial to verify the projection method. We do this with two independent approaches. First, in appendix A, the projection method is used to calculate kinematic transport coefficients for low- $R m$ non-helical shear dynamos, allowing a direct comparison to the kinematic test-field method. The study is carried out for dynamos with a range of positive and negative $\eta_{y x}$ by changing the rotation (see Paper II), and is in a regime where the stochastic- $\alpha$ effect is significant. This ensures that the projection method does not inadvertently capture a property of the dynamo growth rate, rather than the coherent transport coefficients. Second, we verify the calculated transport coefficients are correct a posteriori for the main simulation results $(\S 3.2)$. This is done by solving the mean-field equations (2.4) using the time-dependent transport coefficients $\alpha_{i j}(t)$ and $\eta_{i j}(t)$ calculated with the projection method. Comparison with the mean-field evolution taken directly from the simulation provides a thorough check that the transport coefficients are being calculated correctly, without relying on assumptions about the nature of the dynamo (aside from the mean-field ansatz), or the importance of approximations made to the form of the EMF (i.e. (3.7)).

\subsection{Numerical results - the magnetically driven dynamo}

In this section, we show that small-scale fields arising self-consistently through the small-scale dynamo can drive a coherent large-scale dynamo. To this end, we apply the methods discussed in the previous section to calculate transport coefficients before and after the saturation of the small-scale dynamo. The technique is applied to ensembles of 100 simulations, both with and without Keplerian rotation.

Before continuing, we demonstrate that there is indeed a large-scale dynamo that develops after saturation of the small-scale dynamo. This is shown both in figure 4, which gives the time development of the mean-field energy and turbulent spectra,

\footnotetext{
IIIT This filtering violates the Reynolds averaging rules (specifically $\left\langle\langle g\rangle_{f} h\right\rangle_{f} \neq\langle g\rangle_{f}\langle h\rangle_{f}$, where $\langle\cdot\rangle_{f}$ is the Fourier average). While not technically required for the validity of (3.2) (which relies only on some level of scale separation), the rules are required for the use of the mean-field induction equation ((3.6)) in the first place, since $\langle\boldsymbol{u} \times \boldsymbol{B}\rangle_{f}$ may drive the Fourier-averaged field in addition to $\mathcal{E}$ and $\boldsymbol{U} \times \boldsymbol{B}$ (this is an aliasing effect). To ensure this does not adversely affect results, we have verified that the coefficients are essentially independent of the number of Fourier modes $n$ retained in the projection, up to $n \approx 5$ or 6 .
} 

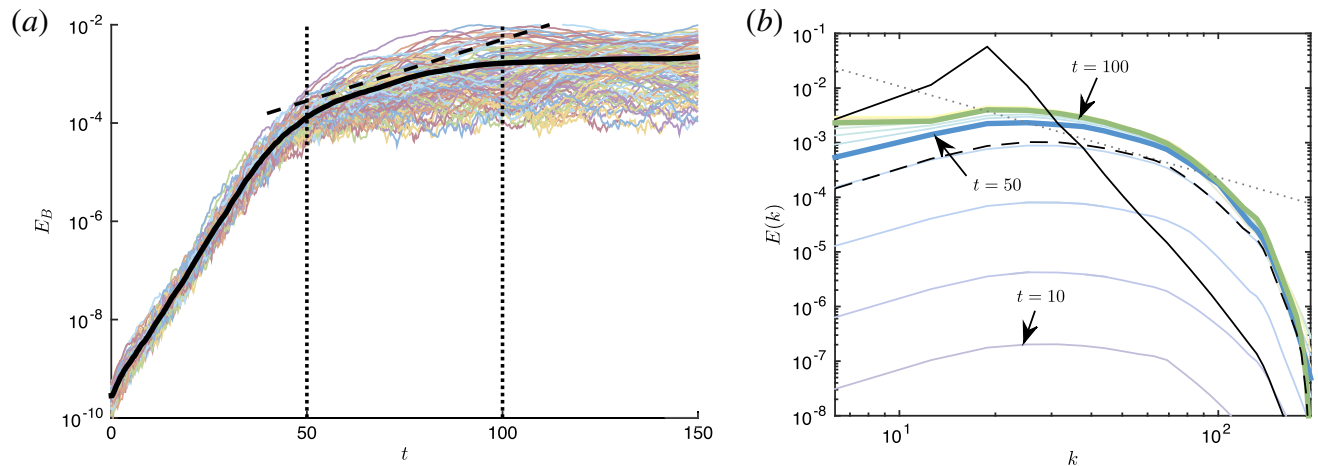

FIgURE 4. (a) Time development of the average mean-field energy $E_{B}=L_{z}^{-1} \int \mathrm{d} z \boldsymbol{B}^{2}$ for the rotating simulation set. Each faded colour curve illustrates a single realization, while the thick black curve shows the mean over all realizations. The dotted vertical lines indicate the saturation of the small-scale dynamo and the nonlinear saturation of the large-scale dynamo (the projection method is applied between these), while the dashed line is simply an approximate fit to the large-scale dynamo growth phase. (b) Shell-averaged turbulent spectra (of $\boldsymbol{B}_{T}$ and $\boldsymbol{U}_{T}$ ) for the rotating simulations shown in $(a)$. The coloured lines (from blue to yellow) illustrate the growth of the magnetic spectrum in time (averaged over all simulations), with the spectra at $t=50, t=100$ and $t=150$ highlighted by thicker lines. The solid black line illustrates the velocity spectrum (peaked at $k \approx 6 \pi$ ), while the dashed line shows the magnetic spectrum from an identical simulation, but without velocity shear $(S=0)$, and averaged in time from $t=50$ to $t=150$. Evidently, as also seen in Yousef et al. (2008a), the second period of large-scale field growth is absent when $S=0$ (note that the velocity spectrum is essentially identical to the case with velocity shear). The dotted line simply illustrates a $k^{-5 / 3}$ spectrum for the sake of clarity. The slight bump in the spectrum at high $k$ is caused by spectral reflection from the grid cutoff; however, since this is well into the exponential fall off and at very low energy we are confident that this does not affect large-scale evolution (note that the spectrum in the few double resolution simulations is essentially identical, aside, at and above the bump itself).

and in figure 5, which illustrates the spatio-temporal evolution of $B_{y}(z, t)$ in several example realizations. From figure 4 , we clearly see the fast growth of the small-scale dynamo until its saturation at $t \approx 50$. (This is observable in figure 4(a), which shows only the mean field, because $\boldsymbol{B}$ is in approximate equipartition with the small scales due to the finite domain.) Following this there is a slower period of growth in only the largest scales of the box (this is $k_{1 z}=\pi$, a factor of six less than the forcing scale), with this saturating around $t=100$ on average. Importantly, this second period of slower growth in the mean field is not present without the mean shear (see figure 4(b), dashed line), despite the velocity spectrum being essentially identical. This illustrates that the shear causes large-scale field generation after saturation, as also noted in Yousef et al. (2008a),

As shown in figures 4(a) and 5, at these parameters, the prevalence of a coherent large-scale dynamo after saturation of the small-scale dynamo varies significantly between realizations. Specifically, it appears that the coherent effect cannot always overcome fluctuations in $\mathcal{E}$ immediately after small-scale saturation, although the dynamo always develops after a sufficiently long time (e.g. figure $5(d)$ near $t=150$ ). This behaviour seems generic when the coherent dynamo is close to its threshold 
(a)

(b)

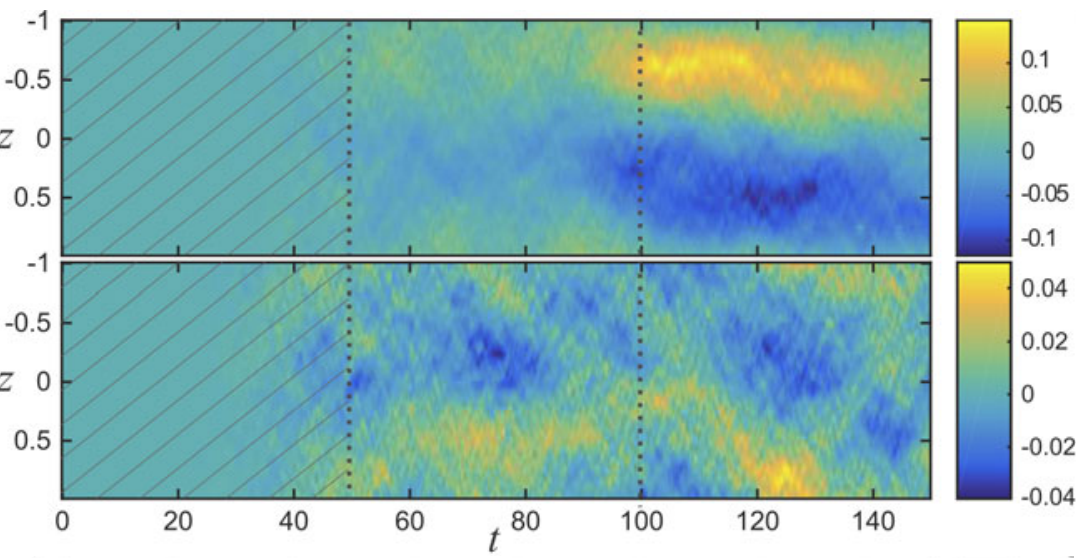

(c)

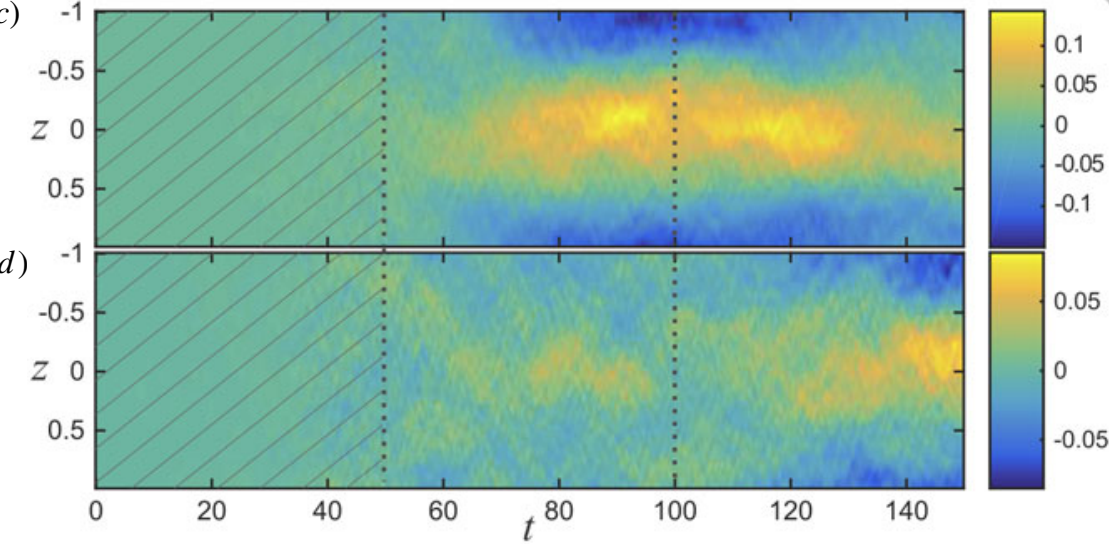

FIGURE 5. Example spatio-temporal $B_{y}$ evolutions for $(a, b)$ non-rotating $(\Omega=0)$, and $(c, d)$ Keplerian rotating $(\Omega=2 / 3)$ driven turbulence (parameters described in the text). The first examples in each case $(a, c)$ show $B_{y}$ when a coherent dynamo develops, while the second examples $(b, d)$ illustrate the case when it is more incoherent. The main factors in distinguishing these are the coherency in phase of $B_{y}$ over some time period and the amplitude at saturation, which is larger in the coherent cases. In general the rotating simulations are substantially more coherent. The hatched area illustrates the region of small-scale dynamo growth. The projection method used to compute transport coefficients (see figure 6) is applied between the dashed lines $(t=50 \rightarrow 100)$.

for excitation, and similar structures were observed at lower $R m$ in Paper II, where forcing in the induction equation was used to create an homogeneous bath of magnetic fluctuations. Nonetheless, despite this variability in the dynamo's qualitative behaviour, measurement of the transport coefficients over the ensemble of simulations illustrates a significant decrease in $\eta_{y x}$ after the magnetic fluctuations reach approximate equipartition with velocity fluctuations at small scales.

At low times, before $t \approx 50$, the kinematic $\alpha$ and $\eta$ are measured using the test-field method, fixing the mean field and calculating $\mathcal{E}$, with no Lorentz force (Brandenburg \& Subramanian 2005; Brandenburg et al. 2008a). Calculations are run from $t=0 \rightarrow 2000$ with the errors estimated through the standard deviation of the mean over 100 segments. Since the small-scale dynamo grows quickly, test fields are reset every $t=5$. After small-scale saturation, we utilize the projection method (\$3.1) to measure 
coefficients directly from the observed mean field and EMF evolution ${ }^{* * *}$. The time window of these measurements has been limited to $t=50 \rightarrow 100$, since growth is seen to stop at $t \approx 100$ in many realizations (see figures 4 and $5 a, c$ ). Since this saturation presumably occurs due to a nonlinear change in the transport coefficients at large $\boldsymbol{B}$ (e.g. a change in sign of $\eta_{y x}$ ), it is important to not include this saturation phase in the measurement of $\eta_{y x}$. As should be expected from figure 5 and due to the short time window, measurements of the transport coefficients after small-scale saturation vary significantly between realization. Nonetheless, an average over the ensemble illustrates a statistically significant change in $\eta_{y x}$ that is consistent with observed behaviour, in both the rotating and non-rotating simulation ensembles.

Figure 6 illustrates the results. In the kinematic phase without rotation, we see $\eta_{y x}=(4.1 \pm 1.6) \times 10^{-4}$, in qualitative agreement with previous studies (Brandenburg et al. 2008a). With rotation, we find $\eta_{y x}=(0.6 \pm 1.2) \times 10^{-4}$, consistent with a reduction in $\eta_{y x}$ due to the $\boldsymbol{\Omega} \times \boldsymbol{J}$ effect (Krause \& Rädler 1980, but note the deviation from the lower- $R m$ case and SOCA result, which predicts negative $\eta_{y x}$ ). After saturation of the small-scale dynamo, $\eta_{y x}=(-0.1 \pm 1.0) \times 10^{-4}$ for the non-rotating case, while $\eta_{y x} \approx-(2.0 \pm 0.8) \times 10^{-4}$ in the rotating case. The reduction of each is the same to within error. Values for the diagonal resistivity are smaller after saturation, which is consistent with the observed decrease in the velocity fluctuation energy (by a factor $\sim 1.4$ ).

The numerical values of $\eta_{x x}$ and $\eta_{y x}$ show that the coherent dynamo is slightly stable on average in the non-rotating case and marginal in the rotating case. However, the coefficients vary significantly between realizations, sometimes yielding larger growth rates, and it is important to check that the observed mean-field evolution has some relation to this variation. This serves two purposes. First, it acts as a check that the projection method is measuring the transport coefficients correctly. Second, it illustrates that those realizations exhibiting the strongest growth are indeed being driven by the shear-current mechanism; that is, they are driven by $\eta_{y x}$ rather than residual variation of $\alpha$ about mean zero. This corroborates the earlier conclusion that the approximately constant phase of $B_{y}(z, t)$ in the development of the dynamo (figure 5) is inconsistent with an $\alpha$ effect.

As stated previously, the method for checking this consistency is to use the measured transport coefficients to solve for the expected evolution of the largest Fourier mode of $B_{i}$ (using (2.4)), comparing this to the observed evolution from the full simulation. Note that we use the time-dependent coefficients $\alpha_{i j}(t)$ and $\eta_{i j}(t)$, rather than the time average that is shown in figure 6 , since this provides much more information about the details of the evolution. The check is carried out for each realization separately, initializing using the mean-field data and filtering transport coefficients in time with a Gaussian filter of width 5 to remove the rapid fluctuations. Results from the first 12 realizations for rotating runs (chosen since the dynamo is stronger than in the non-rotating cases) are shown in figure 7. The agreement is generally good, with qualitatively similar features between calculated and measured evolution in all realizations, and many cases showing quantitative agreement. It seems that in most instances for which there is a substantial divergence between the predicted and observed mean-field evolution, it is due to a slight error building up in $B_{x}$ that subsequently gets amplified enormously due to the $-S B_{x}$ term in the $B_{y}$ equation.

\footnotetext{
***While it would be ideal to measure coefficients before saturation using the projection method for consistency, this is difficult. In particular, the method is hindered by the small-scale dynamo causing the mean-field evolution to be completely overwhelmed by small-scale noise. We explored the possibility of seeding initial conditions with large-scale fields to obtain a short period of kinematic evolution; however, results were inconclusive due to very high levels of noise in the measurements.
} 

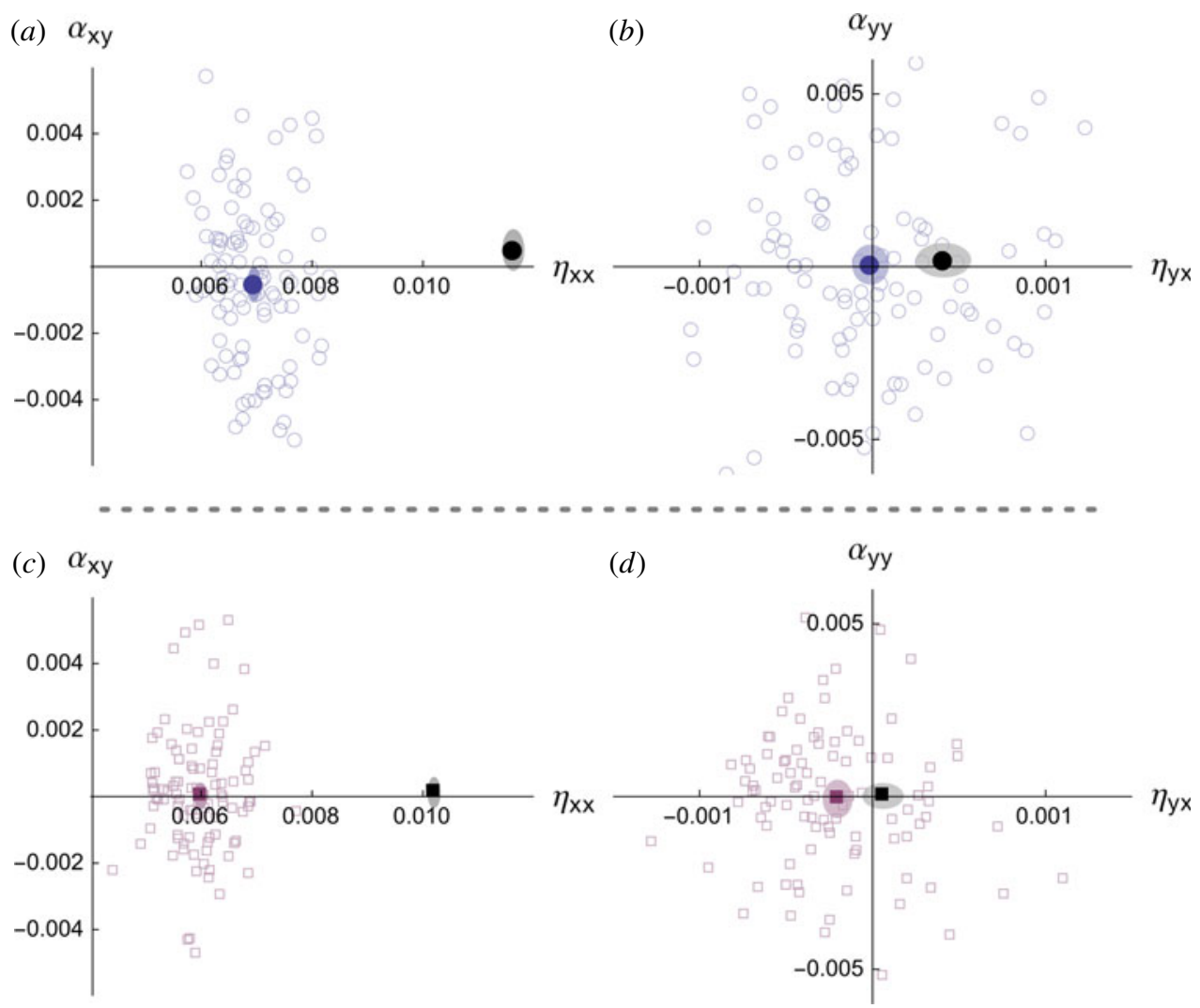

FIGURE 6. Measurements of the turbulent transport coefficients for 100 realizations of the simulations in figure 5; (a) $\eta_{x x}$ coefficients, no rotation, (b) $\eta_{y x}$ coefficients, no rotation, (c) $\eta_{x x}$ coefficients, rotating, (d) $\eta_{y x}$ coefficients, rotating. Unfilled markers in each plot (circles and squares for non-rotating and rotating runs respectively) show coefficients measured from each of the individual realizations, with mean values displayed by solid markers and the shaded regions indicating error in the mean ( 2 standard deviations). Black markers illustrate the kinematic transport coefficients, with grey shaded regions indicating the error. After saturation of the small-scale dynamo, $\eta_{i j}$ is calculated using the projection method, taking the mean from $t=50$ to $t=100$. This limited time window is chosen to avoid capturing the saturation phase of the large-scale dynamo, since $\eta_{i j}$ is presumably modified in this phase. In both methods used to compute transport coefficients, the corresponding $\alpha$ coefficients are also calculated. In all cases these are zero to within error as expected, and the scatter between simulations is of a similar magnitude to that of $\eta_{i j}$ if their different units are accounted for (it is necessary to divide $\alpha$ by a characteristic $k$ value).

In addition to solving for expected evolution using both $\eta_{i j}(t)$ and $\alpha_{i j}(t)$ measurements, we present calculations obtained in an identical way, but with $\alpha_{i j}(t)$ coefficients artificially set to zero. The purpose of this analysis is to examine the degree to which the dynamo is driven by $\eta_{y x}$, rather than variation in $\alpha$ about its mean of zero. Through a comparison of the curves with and without $\alpha_{i j}$ it is clear that in many realizations of the rotating simulation set, the dynamo is primarily driven by $\eta_{y x}$, as shown by the agreement between dashed and dotted curves. Furthermore, the 

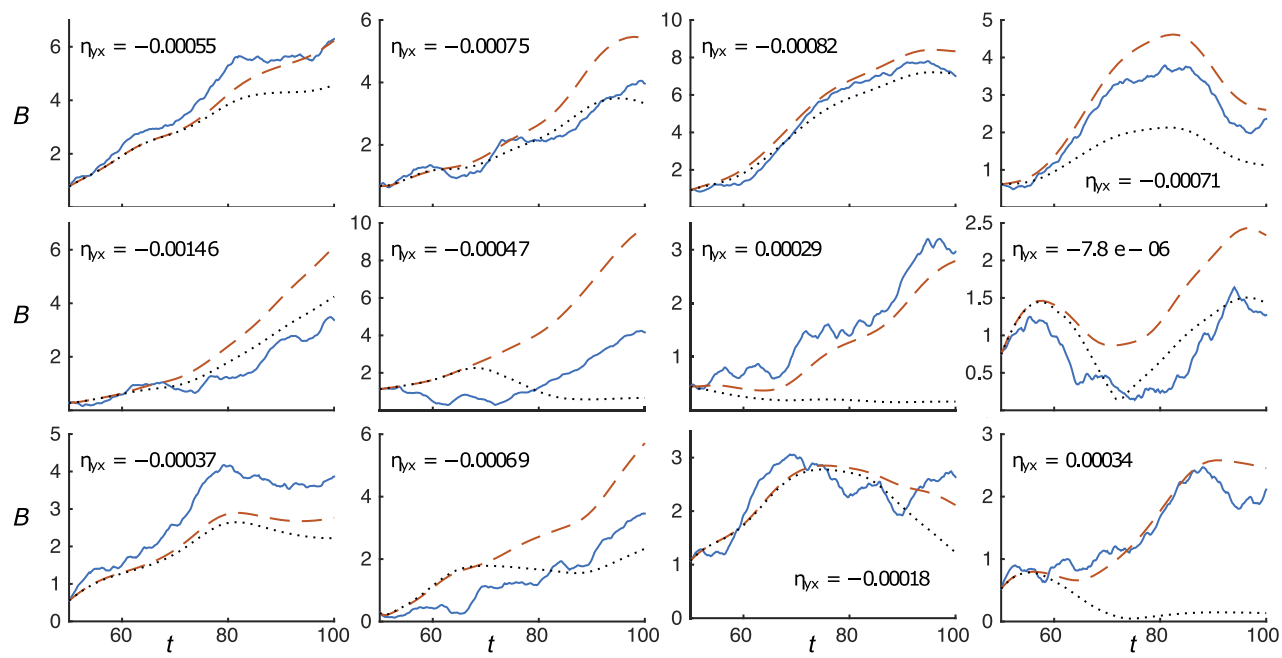

FIGURE 7. Evolution of the mean-field magnitude for the first 12 of the ensemble of rotating simulations discussed in the manuscript. Here $B\left(\left|\hat{B}_{x}^{1}\right|^{2}+\left|\hat{B}_{y}^{1}\right|^{2}\right)^{1 / 2}$ is the mean-field magnitude, where $\hat{B}_{i}^{1}$ is the largest scale Fourier mode of $B_{i}$. In each plot the solid blue curve shows data taken from the simulation. The dashed red curve shows the corresponding expected evolution, using the smoothed calculated values of the transport coefficients (see text). Finally, the dotted black curve illustrates the expected evolution, artificially setting all $\alpha$ coefficients to zero. We list the measured mean of $\eta_{y x}$ in each plot to show that higher absolute values (i.e. more negative values) do generally lead to substantially more growth of the mean field as expected for a coherent dynamo. For reference, at the measured $\eta_{x x} \approx 0.006$, the coherent dynamo is unstable below $\eta_{y x}=-0.00036$.

mean of $\eta_{y x}$ over the time interval (printed on each subfigure; these are taken from figure 6) agrees nicely with the observed behaviour. That is, large negative values for $\eta_{y x}$ correspond to those realizations with both strong dynamo growth and good agreement between evolution with and without $\alpha$. In contrast, realizations with lower absolute values of $\eta_{y x}$ (i.e. values for which the dynamo is stable) either grow very little or diverge substantially between evolution with and without $\alpha$. This shows that sometimes, for realizations in which the magnetic shear-current effect is weaker, a stochastic $\alpha$ effect is the primary driver. A similar examination of the non-rotating case shows that coherent dynamo growth is much less prevalent. In particular, while the agreement between the true and calculated evolution is satisfactory (similar to figure 7), there is generally much less mean-field growth and larger differences with calculations for which $\alpha_{i j}$ is artificially set to zero. Since in most realizations $\eta_{y x}$ is larger than the threshold at which the coherent dynamo becomes unstable even after the decrease due to magnetic fluctuations, this is not surprising.

We thus conclude that small-scale magnetic fluctuations act to make $\eta_{y x}$ more negative, and that in some realizations (or after a sufficiently long time period) a coherent large-scale dynamo develops as a result. This demonstrates that magnetic fluctuations, excited by small-scale dynamo action, can drive large-scale magnetic field generation. The consistency of the numerical simulations with theoretical expectations, as well as the general agreement of measured transport coefficients with observed mean-field evolution, give us confidence that the observed large-scale 
dynamo is indeed a coherent effect. The mechanism is the magnetic shear-current effect, arising through the contribution of magnetic fluctuations to the off-diagonal turbulent resistivity $\eta_{y x}$ in the presence of large-scale shear flow.

The most significant limitation of the studies presented in this section is the relatively low Reynolds numbers, which were chosen to be slightly below the transition to self-sustaining turbulence (in the absence of driving noise), as well as for computational reasons (since an ensemble of simulations was required). Specifically, the dynamo is likely far from any asymptotic regime at high $R e$ and $R m$. This is almost certainly true for both the small-scale dynamo and its saturation ${ }^{\dagger \dagger}$, as well as for the large-scale magnetic shear-current effect itself. Further, the diffusion time scale of the large-scale modes is $t \approx\left(\bar{\eta}\left(2 \pi / L_{z}\right)^{2}\right) \approx 200$, which is only an order of magnitude different from the time scale of growth of the large-scale field $(t \approx 30$, as can be seen from figure 7). The same is true of the separation between the turbulent forcing scale $k_{f} \approx 19$, and that of the large-scale field $k_{1}=2 \pi / L_{z}=\pi$. While such limitations are hardly unique in the dynamo literature, it is obviously pertinent to undertake future studies at much higher resolutions. Further discussion of some of the difficulties involved with truly understanding the astrophysical relevance of the magnetic shear-current effect is given in the next section.

\section{Discussion and conclusions}

This paper has revolved around exploration of the 'magnetic shear-current effect' as a viable mechanism to drive large-scale dynamos in non-helical shear flows. The suggestion is that a bath of homogeneous non-helical magnetic fluctuations, influenced by the velocity shear, can cause a large-scale dynamo instability through an off-diagonal turbulent resistivity, even if there is no $\alpha$ effect. More specifically, in response to a large-scale azimuthal magnetic field $B_{y}$, a bath of magnetic fluctuations will produce an azimuthal electromotive force $\mathcal{E}_{y}$, proportional to $\partial_{z} B_{y}$. This $\mathcal{E}_{y}$ causes the generation of a radial magnetic field, which in turn amplifies the azimuthal field through stretching by the mean flow (the $\Omega$ effect), resulting in a dynamo instability. The effect rests crucially on the sign of the proportionality between $\mathcal{E}_{y}$ and $\partial_{z} B_{y}$ (termed $\left.\eta_{y x}\right)$ - if the product $\eta_{y x}(\nabla \times \boldsymbol{U})_{z}$ is negative, the induced radial field will act to damp, rather than amplify, the azimuthal field.

The physical picture for the magnetic shear-current effect - how magnetic fluctuations can interact with velocity shear and a large-scale field gradient to produce an $\mathcal{E}$ of the required direction - is somewhat different from dynamo mechanisms described in previous literature (see, for example, Brandenburg \& Subramanian 2005, Yokoi 2013). In particular, it relies on the pressure response of the fluid to the Maxwell stress $\boldsymbol{B}_{T} \cdot \boldsymbol{\nabla} \boldsymbol{B}_{T}$. The basic effect arises because $\boldsymbol{b} \cdot \nabla \boldsymbol{B}$ creates $\boldsymbol{B}$-directed velocity perturbations from magnetic perturbations in the direction of magnetic shear (i.e. the $\boldsymbol{\nabla}$ direction). This implies that any variation of the $\boldsymbol{b}$ perturbation along the $\boldsymbol{B}$ direction will create a velocity perturbation with non-zero divergence, leading to a significant pressure response. Without velocity shear, the response is well known and fundamental for turbulent diffusion; it exactly cancels another term and causes the contribution to the turbulent mean-field resistivity from magnetic fluctuations to vanish (this is also known as the absence of $\beta$-quenching; Gruzinov \& Diamond 1994). In the presence of velocity shear, a secondary pressure response, arising due to the

\footnotetext{
$\dagger \dagger$ The critical $R m$ for onset of the small-scale dynamo with this forcing is $R m_{C} \approx 1100$, so we are well away from where one might expect the growth rate or saturated field level to converge (Tobias \& Cattaneo 2015).
} 
stretching of the primary pressure response by the mean shear, causes perpendicular velocity fluctuations that are correlated with the original magnetic fluctuations. The resulting EMF is in the required direction to generate a $\boldsymbol{B}$ that is stretched by the shear flow, enhancing the mean field that caused the effect in the first place. Thus, a mean-field dynamo instability can ensue at sufficiently long wavelength.

Why is magnetic shear-current mechanism interesting? We would like to give two answers to this question: the first relates generally to dynamo theory, the second to the specific case of the dynamo seen in simulations of turbulence in accretion disks (the MRI dynamo).

General mean-field dynamo theory. Much of mean-field dynamo theory in recent years has focused on the issue of $\alpha$ quenching (Kulsrud \& Anderson 1992; Gruzinov \& Diamond 1994). This is specifically related to the adverse influence of small-scale magnetic fields on large-scale dynamo action. Since small-scale dynamos grow faster than large-scale fields above moderate Reynolds numbers, large-scale dynamos may always have to grow on a bath of small-scale magnetic fluctuations (Cattaneo \& Hughes 2009, but see also Tobias \& Cattaneo 2014). With this in mind, the magnetic shear-current effect is the first suggestion (of which we are aware) for a large-scale dynamo driven by small-scale magnetic fluctuations (although quenching of the turbulent resistivity can lead to a dynamo with spatial variation of transport

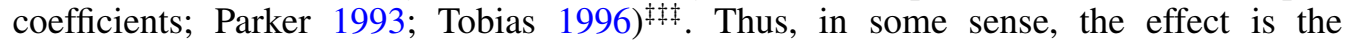
inverse of dynamo quenching; rather than magnetic fluctuations overwhelming a desirable kinematic effect, mean-field growth starts after small-scale dynamo saturation, driven by the small-scale field itself. In this work, we have given an example of this interesting behaviour through targeted numerical experiments. These illustrate that the magnetic fluctuations resulting from saturation of the small-scale dynamo cause a significant decrease (and in some cases, a sign change) of the crucial $\eta_{y x}$ transport coefficient, which can in turn drive a large-scale dynamo. Study of such magnetic dynamos in direct numerical simulations is confounded by the very short period of exponential growth that can be observed (in contrast with kinematic shear dynamos; Yousef et al. 2008b; Paper II), and more work is needed to better assess regimes where the effect might be dominant, or even if it continues to operate at very high Reynolds numbers. Nonetheless, it is an interesting possibility that may find application across a wide variety of astrophysical objects.

The MRI dynamo. The central regions of accretion disks are both unstratified and lack a source of net kinetic or magnetic helicity, implying that an $\alpha$ effect is not possible. In addition, a variety of authors have found from simulation and theory that the crucial $\eta_{y x}$ is of the wrong sign for a kinematic non-helical shear dynamo (Rädler \& Stepanov 2006; Rüdiger \& Kitchatinov 2006; Singh \& Sridhar 2011; Paper II). What then is the cause of the apparent large-scale dynamo seen in simulations? While there is the possibility that it is driven by fluctuations in the $\alpha$ coefficients (Vishniac \& Brandenburg 1997; Vishniac 2009), we would argue that the magnetic shear-current effect is a more likely candidate: MRI simulations exhibit stronger magnetic than kinetic fluctuations, the Keplerian rotation is favourable for dynamo growth, the

\footnotetext{
$\ddagger \ddagger$ The magnetic $\alpha$ effect can certainly drive a mean field in isolation. The key point is that its sign is opposite to that of the kinetic effect, and the small-scale dynamo grows such that the two effects cancel. While it may be possible that instabilities would cause a magnetic $\alpha$ effect to overwhelm the kinematic one (for instance, the MRI in the presence of stratification, Gressel 2010; Park \& Blackman 2012), this remains unclear. In contrast, the magnetic shear-current effect has a fixed sign, arising from the non-helical part of the fluctuations.
} 
velocity shear is obviously important, and the nonlinear behaviour of the effect bears strong similarities to mean-field dynamics in unstratified MRI simulations. In addition, the basic importance of $\eta_{y x}$ in the MRI dynamo has been concluded from nonlinear simulation (Lesur \& Ogilvie 2008b) and perturbative calculations of the evolution of MRI modes (Lesur \& Ogilvie 2008a). Our suggestion that small-scale magnetic fields are in fact the primary driver thus ties together formal mean-field dynamo theory with these studies and explains the special importance of strong magnetic fluctuations in MRI turbulence and dynamo.

Some of the most compelling evidence that the magnetic shear-current effect is indeed responsible for the unstratified MRI dynamo comes from statistical simulation of the saturation of MRI turbulence (Squire \& Bhattacharjee 2015a). Statistical simulation (Tobias, Dagon \& Marston 2011b, Farrell \& Ioannou 2012) involves formulating equations for statistics of the small-scale fields $\boldsymbol{u}$ and $\boldsymbol{b}$ in the mean fields $(\boldsymbol{U}$ and $\boldsymbol{B})$, and solving these, rather than a single turbulent realization. Importantly for the shear dynamo, this completely eliminates the possibility of a stochastic- $\alpha$ effect, since the $\mathcal{E}$ that drives $\boldsymbol{B}$ is calculated directly from fluctuation statistics. Coupled with the fact that the kinematic effect is too weak to explain the dynamo (Paper II), it is clear that the magnetic shear-current effect is the only possible field generation mechanism in these calculations. Despite this, the agreement with nonlinear simulation is very good (see figure 2 of Squire \& Bhattacharjee 2015a). Most important is the observed strong increase in the saturated mean $\boldsymbol{B}$ field, and consequently in the turbulent angular momentum transport, as the magnetic Prandtl number is increased at fixed $R e$. This counterintuitive trend has been the source of much discussion in the MRI turbulence literature (see, for example, Fromang et al. 2007, Lesur \& Longaretti 2007, Meheut et al. 2015). The considerations above illustrate that it is, at least in part, a consequence of the $P m$ dependence of the saturation of the magnetic shear-current effect.

Looking past the unstratified MRI dynamo, we might wonder about other applications of the magnetic shear-current effect. Large-scale velocity shear is inescapable in the universe due to the influence of gravity, while the generic instability of small-scale dynamo at large Reynolds numbers implies that plasma turbulence should always be accompanied by small-scale magnetic fluctuations in near equipartition with velocity fluctuations (Schekochihin et al. 2007). However, the simulations discussed in $\S 3$ are intended to illustrate that the magnetic shear-current effect is possible, not necessarily that it should be important in every situation. Unfortunately, estimating the relevance of the effect in astrophysical scenarios in any detail requires more knowledge about its dependence on physical parameters - particularly the Reynolds numbers (and magnetic Prandtl number). There are numerous complicating factors that will arise in estimating these dependencies. Most obvious is the variation of transport coefficients themselves (especially $\eta_{y x}$ ) for a given level of magnetic fluctuations. While it is certainly encouraging that a variety of different methods agree on the sign of $\eta_{y x}$, most results are truly valid only at low Reynolds numbers ${ }^{\S \S \S}$. More subtly, the relevance of the effect could depend significantly on the saturation level of the small-scale dynamo, which would be especially important if the kinematic shear-current effect has the incorrect sign for dynamo action $\left(\left(\eta_{y x}\right)_{u}\right.$ may change sign with Reynolds number; see Brandenburg et al. 2008a). This saturation level presumably depends on Pm, but may also change

\footnotetext{
$\S \S$ The spectral $\tau$ approximation (which predicts $\left(\eta_{y x}\right)_{b}<0$ and $\left|\left(\eta_{y x}\right)_{b}\right| \gg\left|\left(\eta_{y x}\right)_{u}\right|$; Rogachevskii \& Kleeorin 2004) is nominally valid at high Reynolds number, but its accuracy and reliability remain unclear (see, for example, Rädler \& Rheinhardt 2007).
} 
under the influence of velocity shear (at least at the larger of the small scales), an effect that may become significant only at very high Reynolds numbers (Cattaneo \& Tobias 2014; Tobias \& Cattaneo 2014). Finally, magnetic helicity and its transport are a cornerstone of modern dynamo theory (Vishniac \& Cho 2001; Field \& Blackman 2002), but have not been explored in our work thus far due to the focus on the linear phases of the dynamo instability. Such effects will be important to consider in future studies of the saturation and nonlinear evolution of magnetic shear-current dynamos (Rogachevskii, Kleeorin \& Liverts 2006). Overall, given the general difficulty of even measuring growth rates for magnetically driven large-scale dynamos, it seems that the magnetic shear-current effect will provide a variety of rich and interesting avenues for future exploration.

\section{Acknowledgements}

The authors would like to thank J. Krommes, J. Goodman, H. Ji, G. Hammett, and A. Schekochihin for enlightening discussion and useful suggestions, as well as G. Lesur for distribution of the SNOOPY code. J.S. acknowledges the generous support of a Burke Fellowship and the Sherman Fairchild Foundation at Caltech, as well as a Procter Fellowship at Princeton University. This work was funded by US Department of Energy grant no. DE-AC02-09-CH11466 and computations were carried out on the Dawson cluster at PPPL.

\section{Appendix A. Verification of the projection method: low- $R m$ shear dynamo}

In this appendix, we verify that the projection method discussed in $\S 3.1$ recovers the correct transport coefficients for low- $R m$ shear dynamos, similar to those studied in Paper II and Yousef et al. $(2008 a, b)$. The primary advantage of testing the method in this parameter regime is that there is no small-scale dynamo and simulations exhibit a very long kinematic growth period over which the small-scale velocity field is unaffected by the magnetic field. It is thus straightforward to compare results obtained with the projection method to those using the test-field method, where the only the fluctuating part of the induction equation is solved.

The simulations are carried out in the same numerical set-up as used in the main text, but at $R e=R m=100$ in shearing boxes of dimension $\left(L_{x}, L_{y}, L_{z}\right)=(1,1,8)$. The large $L_{z}$ is chosen to allow for a long mean-field wavelength and thus enhance the dynamo instability (Yousef et al. 2008a). The velocity field is forced at $k=6 \pi$ to a level $u_{r m s} \approx 0.8$, using the same non-helical $\sigma_{u}$ as detailed in the main text. Keeping $S=2$, we present cases that are non-rotating, $\Omega=0$, as well as $\Omega=4 / 3$ (Keplerian), and $\Omega=4$, with the rotation added through the mean Coriolis force. As discussed in Paper II, this change in $\Omega$ causes $\eta_{y x}$ to change sign due to the $\Omega \times J$ (or Rädler) effect. We have run 10 simulations at each parameter set from $t=0$ to $t=1000$, although the rotating cases saturate earlier $(\Omega=4 / 3$ at $t \approx 900, \Omega=4$ at $t \approx 500)$ due to faster dynamo growth. Note that the ratio of $B_{y}$ to $B_{x}$ in these simulations $(\sim 10 \rightarrow 30$ during growth) is somewhat higher than that for the magnetically driven dynamos studied in $\S 3.2$; thus, if anything, one might expect larger systematic errors in these simulations than the estimates given in $\$ 3.1$.

Test-field calculations are conducted as discussed in $\S 3.1$. Due to the lack of a small-scale dynamo, the $\boldsymbol{b}$ fluctuations quickly reach a steady state, and an average of $\mathcal{E}$ is taken over $t=0 \rightarrow 1600$ to obtain $\eta_{x x}$ and $\eta_{y x}$. Errors are obtained through the standard deviation of the mean after dividing the data set into 100 bins ( \pm values indicate the $95 \%$ confidence interval). Results from the test-field method, which we 
(a)

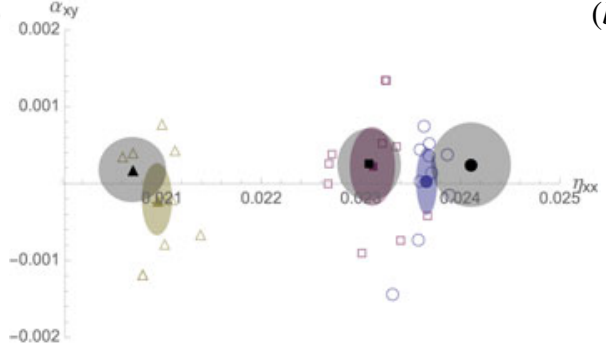

(b)

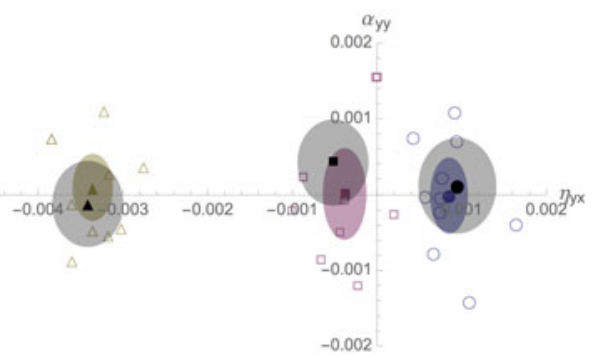

FIGURE 8. Measured transport coefficients for $R e=R m=100$ shearing box simulations (as described in the text). (a) $\eta_{x x}$, (b) $\eta_{y x}$. Squares, circles and triangles show $\Omega=0, \Omega=4 / 3$ and $\Omega=4$ respectively, with the hollow markers illustrating those measured from each simulation. The mean of these measurements is shown by the solid coloured marker, with its error shown with the shaded circle (calculated from the standard deviation). Test-field method results, against which least squares results should be compared, are illustrated by black markers, with the shaded area showing the error in these measurements. (We have also included the measured $\alpha$ values here, although in all cases these are zero to within error.)

consider as the reference values against which to compare coefficients obtained using the fitting method, are illustrated in figure 8 in black. These values are comparable (in the ratio of $\eta_{x x}$ to $\eta_{y x}$ ) to those obtained in previous work for the non-rotating case (Brandenburg et al. 2008a), as well as exhibiting the expected trends (Rädler \& Stepanov 2006; Paper II). In all cases the test fields measured $\alpha_{i j}$ are zero to within error (see figure 8).

Results obtained by using the projection method on the self-consistent MHD simulations are also illustrated in figure 8 . The transport coefficients $\left(\alpha_{x y}, \alpha_{y y}, \eta_{x x}, \eta_{y x}\right)$ are measured as described in $\$ 3.1$ for the duration of each simulation, excluding times after which the dynamo has saturated. Because of the long averaging time in comparison to the $R m=2000$ measurements presented in $\S 3.2$, the spread of values between different simulations is quite small. It is seen that the measured coefficients agree with the test-field calculations to within error margins in all cases. The largest discrepancy is in $\eta_{x x}$ at $\Omega=0$, which may be related to the vorticity dynamo (i.e. mean flow generation) that develops without rotation (the difference is still only of the order of $1 \%$ ). Note that a growing dynamo is observed in all of the self-consistent simulations, and at $\Omega=0$ this is purely due to a stochastic- $\alpha$ effect (as discussed in Paper II), since the measured transport coefficients indicate the dynamo should be stable. We can thus be sure that the fitting method is not somehow measuring a property of the dynamo growth rate rather than coherent transport coefficients.

\section{REFERENCES}

AvinASH, K. 1991 Dynamo effect and current drive due to magnetic fluctuations in sheared magnetic field. Phys. Fluids B 3, 2150.

Bhattacharjee, A. \& Yuan, Y. 1995 Self-consistency constraints on the dynamo mechanism. Astrophys. J. 449, 739-744.

Blackman, E. \& Field, G. 2002 New dynamical mean-field dynamo theory and closure approach. Phys. Rev. Lett. 89, 265007. 
Boldyrev, S., Cattaneo, F. \& Rosner, R. 2005 Magnetic-field generation in helical turbulence. Phys. Rev. Lett. 95, 255001.

Brandenburg, A., Nordlund, A., Stein, R. F. \& Torkelsson, U. 1995 Dynamo-generated turbulence and large-scale magnetic fields in a Keplerian shear flow. Astrophys. J. 446, $741-754$.

Brandenburg, A., RÄDler, K. H., Rheinhardt, M. \& KÄPylä, P. J. 2008 a Magnetic diffusivity tensor and dynamo effects in rotating and shearing turbulence. Astrophys. J. 676, 740-751.

Brandenburg, A., RÄdler, K.-H., Rheinhardt, M. \& Subramanian, K. $2008 b$ Magnetic quenching of $\alpha$ and diffusivity tensors in helical turbulence. Astrophys. J. 687, L49-L52.

Brandenburg, A. \& Sokoloff, D. 2002 Local and nonlocal magnetic diffusion and alpha-effect tensors in shear flow turbulence. Geophys. Astrophys. Fluid Dyn. 96, 319-344.

Brandenburg, A. \& Subramanian, K. 2005 Astrophysical magnetic fields and nonlinear dynamo theory. Phys. Rep. 417, 1-209.

Cattaneo, F. \& Hughes, D. W. 2009 Problems with kinematic mean field electrodynamics at high magnetic Reynolds numbers. Mon. Not. R. Astron. Soc. 395, L48-L51.

Cattaneo, F. \& Tobias, S. M. 2014 On large-scale dynamo action at high magnetic Reynolds number. Astrophys. J. Lett. 789, 70-80.

Ebrahimi, F. \& Bhattacharjee, A. 2014 Helicity-flux-driven $\alpha$ effect in laboratory and astrophysical plasmas. Phys. Rev. Lett. 112, 125003.

FARrell, B. F. \& IOANnOU, P. J. 2012 Dynamics of streamwise rolls and streaks in turbulent wall-bounded shear flow. J. Fluid Mech. 708, 149-196.

Field, G. B. \& Blackman, E. G. 2002 Dynamical quenching of the $\alpha^{2}$ dynamo. Astrophys. $J$. 572, 685-692.

Fromang, S., Papaloizou, J., Lesur, G. \& Heinemann, T. 2007 MHD simulations of the magnetorotational instability in a shearing box with zero net flux. II. The effect of transport coefficients. Astron. Astrophys. 476, 1123-1132.

GRESSEL, O. 2010 A mean-field approach to the propagation of field patterns in stratified magnetorotational turbulence. Mon. Not. R. Astron. Soc. 405, 41-48.

Gressel, O., Bendre, A. \& Elstner, D. 2013 On the magnetic quenching of mean-field effects in supersonic interstellar turbulence. Mon. Not. R. Astron. Soc. 429, 967-972.

Gressel, O. \& Pessah, M. E. 2015 Characterizing the mean-field dynamo in turbulent accretion disks. Astrophys. J. 810 (1), 59-73.

Gruzinov, A. V. \& Diamond, P. H. 1994 Self-consistent theory of mean-field electrodynamics. Phys. Rev. Lett. 72, 1651-1653.

Hawley, J. F., Gammie, C. F. \& Balbus, S. A. 1996 Local three-dimensional simulations of an accretion disk hydromagnetic dynamo. Astrophys. J. 464, 690-703.

Heinemann, T., Mcwilliams, J. C. \& Schekochinin, A. A. 2011 Large-scale magnetic field generation by randomly forced shearing waves. Phys. Rev. Lett. 107, 255004.

Hubbard, A., Del Sordo, F., Käpylä, P. J. \& Brandenburg, A. 2009 The $\alpha$ effect with imposed and dynamo-generated magnetic fields. Mon. Not. R. Astron. Soc. 398, 1891-1899.

Kowal, G., Otmianowska-Mazur, K. \& Hanasz, M. 2005 Calculation of dynamo coefficients in cosmic-ray driven dynamo experiment. In The Magnetized Plasma in Galaxy Evolution, pp. 171-176. Jagiellonian University.

Krause, F. \& RÄDleR, K. H. 1980 Mean-field Magnetohydrodynamics and Dynamo Theory. Pergamon.

Kulsrud, R. M. \& Anderson, S. W. 1992 The spectrum of random magnetic fields in the mean field dynamo theory of the galactic magnetic field. Astrophys. J. 396, 606-630.

Lesur, G. \& Longaretti, P. Y. 2007 Impact of dimensionless numbers on the efficiency of magnetorotational instability induced turbulent transport. Mon. Not. R. Astron. Soc. 378, $1471-1480$.

Lesur, G. \& Ogilvie, G. I. $2008 a$ Localized magnetorotational instability and its role in the accretion disc dynamo. Mon. Not. R. Astron. Soc. 391, 1437-1450.

Lesur, G. \& OGilvie, G. I. $2008 b$ On self-sustained dynamo cycles in accretion discs. Astron. Astrophys. 488, 451-461. 
Meheut, H., Fromang, S., Lesur, G., Joos, M. \& Longaretti, P.-Y. 2015 Angular momentum transport and large eddy simulations in magnetorotational turbulence: the small $\mathrm{Pm}$ limit. Astron. Astrophys. 579, A117.

Mitra, D. \& Brandenburg, A. 2012 Scaling and intermittency in incoherent $\alpha$-shear dynamo. Mon. Not. R. Astron. Soc. 420, 2170-2177.

Moffatt, H. K. 1978 Magnetic Field Generation in Electrically Conducting Fluids. Cambridge University Press.

Park, K. \& Blackman, E. G. 2012 Simulations of a magnetic fluctuation driven large-scale dynamo and comparison with a two-scale model. Mon. Not. R. Astron. Soc. 423, 2120-2131.

PARKER, E. N. 1993 A solar dynamo surface wave at the interface between convection and nonuniform rotation. Astrophys. J. 408, 707-719.

Racine, É., Charbonneau, P., Ghizaru, M., Bouchat, A. \& Smolarkiewicz, P. K. 2011 On the mode of dynamo action in a global large-eddy simulation of solar convection. Astrophys. J. 735 (1), 46-67.

RÄDler, K. H., Kleeorin, N. \& RogachevskiI, I. 2003 The mean electromotive force for MHD turbulence: the case of a weak mean magnetic field and slow rotation. Geophys. Astrophys. Fluid Dyn. 97, 249-268.

RÄDLER，K.-H. \& RHEINHARDT, M. 2007 Mean-field electrodynamics: critical analysis of various analytical approaches to the mean electromotive force. Geophys. Astrophys. Fluid Dyn. 101, $117-154$.

RÄDleR, K.-H. \& Stepanov, R. 2006 Mean electromotive force due to turbulence of a conducting fluid in the presence of mean flow. Phys. Rev. E 73, 056311.

RheinhardT, M. \& BRAndenburg, A. 2010 Test-field method for mean-field coefficients with MHD background. Astron. Astrophys. 520, 28-45.

RogachevskiI, I. \& KLEeORIN, N. 2003 Electromotive force and large-scale magnetic dynamo in a turbulent flow with a mean shear. Phys. Rev. E 68, 036301.

RogacheVskiI, I. \& KLEeORIN, N. 2004 Nonlinear theory of a 'shear-current' effect and mean-field magnetic dynamos. Phys. Rev. E 70, 046310.

RogacheVskit, I., KleEorin, N. \& LiVerTs, E. 2006 Nonlinear shear-current dynamo and magnetic helicity transport in sheared turbulence. Geophys. Astrophys. Fluid Dyn. 100, 537-557.

RÜdiger, G. \& Kitchatinov, L. L. 2006 Do mean-field dynamos in nonrotating turbulent shearflows exist? Astron. Nachr. 327, 298-303.

Scherochinin, A., Cowley, S., Taylor, S., Hammett, G., Maron, J. \& Mcwilliams, J. 2004 Saturated state of the nonlinear small-scale dynamo. Phys. Rev. Lett. 92, 084504.

Schekochihin, A. A., Iskakov, A. B., Cowley, S. C., Mcwilliams, J. C., Proctor, M. R. E. \& Yousef, T. A. 2007 Fluctuation dynamo and turbulent induction at low magnetic Prandtl numbers. New J. Phys. 9, 300-324.

Schrinner, M., RÄDler, K. H, Schmitt, D, Rheinhardt, M \& Christensen, U 2005 Mean-field view on rotating magnetoconvection and a geodynamo model. Astron. Nachr. 326, 245-249.

Simard, C., Charbonneau, P. \& Bouchat, A. 2013 Magnetohydrodynamic simulation-driven kinematic mean-field model of the solar cycle. Astrophys. J. 768 (1), 16.

Singh, N. K. \& JINGADE, N. 2015 Numerical studies of dynamo action in a turbulent shear flow. I. Astrophys. J. 806 (1), 118-127.

Singh, N. K. \& SRIDHAR, S. 2011 Transport coefficients for the shear dynamo problem at small Reynolds numbers. Phys. Rev. E 83, 056309.

Squire, J. \& BhATtACHARJEe, A. $2015 a$ Statistical simulation of the magnetorotational dynamo. Phys. Rev. Lett. 114, 085002.

Squire, J. \& Bhattacharjee, A. $2015 b$ Generation of large-scale magnetic fields by small-scale dynamo in shear flows. Phys. Rev. Lett. 115 (17), 175003.

Squire, J. \& BhattacharJee, A. 2015c Coherent nonhelical shear dynamos driven by magnetic fluctuations at low Reynolds numbers. Astrophys. J. 813 (1), 52-66. 
Squire, J. \& Bhattacharjee, A. 2015d Electromotive force due to magnetohydrodynamic fluctuations in sheared rotating turbulence. Phys. Rev. E 92 (5), 053101.

Subramanian, K. \& BRANDEnburg, A. 2004 Nonlinear current helicity fluxes in turbulent dynamos and alpha quenching. Phys. Rev. Lett. 93, 205001.

Tobias, S. M. 1996 Diffusivity quenching as a mechanism for Parker's surface dynamo. Astrophys. J. 467, 870-880.

Tobias, S. M. \& CATtaneo, F. 2013 On the measurement of the turbulent diffusivity of a large-scale magnetic field. J. Fluid Mech. 717, 347-360.

Tobias, S. M. \& CATtaneo, F. 2014 Shear-driven dynamo waves at high magnetic Reynolds number. Nature 497, 463-465.

Tobias, S. M. \& CAtTaneo, F. 2015 The electromotive force in multi-scale flows at high magnetic Reynolds number. J. Plasma Phys. 81 (6), 395810601.

Tobias, S. M., CattaneO, F. \& BRUmmell, N. H. $2011 a$ On the generation of organized magnetic fields. Astrophys. J. 728, 153-160.

Tobias, S. M., Dagon, K. \& Marston, J. B. $2011 b$ Astrophysical fluid dynamics via direct statistical simulation. Astrophys. J. 727, 127-138.

URPIN, V. 1999 Mean electromotive force and dynamo action in a turbulent flow. Astron. Astrophys. 347, L47-L50.

URPIN, V. 2002 Mean electromotive force in turbulent shear flow. Phys. Rev. E 65, 026301.

VishniaC, E. T. 2009 The saturation limit of the magnetorotational instability. Astrophys. J. 696, $1021-1028$.

Vishniac, E. T. \& Brandenburg, A. 1997 An incoherent alpha-omega dynamo in accretion disks. Astrophys. J. 475, 263-274.

VishniAC, E. T. \& CHO, J. 2001 Magnetic helicity conservation and astrophysical dynamos. Astrophys. J. 550, 752-760.

Yokol, N. 2013 Cross helicity and related dynamo. Geophys. Astrophys. Fluid Dyn. 107, 114-184. Yousef, T. A., Heinemann, T., Rincon, F., Schekochihin, A. A., Kleeorin, N., Rogachevskit, I., Cowley, S. C. \& MCwilliams, J. C. $2008 a$ Numerical experiments on dynamo action in sheared and rotating turbulence. Astron. Nachr. 329, 737-749.

Yousef, T. A., Heinemann, T., Schekochihin, A. A., Kleeorin, N., Rogachevskit, I., Iskakov, A. B., Cowley, S. C. \& Mcwilliams, J. C. $2008 b$ Generation of magnetic field by combined action of turbulence and shear. Phys. Rev. Lett. 100, 184501.

\section{Jonathan Squire}

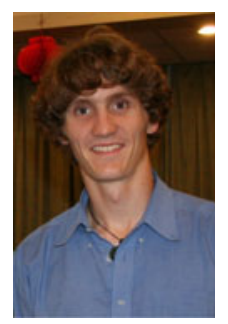

Jonathan Squire carried out his undergraduate degree in physics at Otago University in New Zealand, before going to Princeton on a Fulbright Science and Technology Fellowship in 2010 to start a $\mathrm{PhD}$ at the Princeton Plasma Physics Lab. Here he worked on a variety of projects, mainly related to theoretical aspects of plasma physics and fusion energy, before starting thesis work with Amitava Bhattacharjee on turbulence and magnetic field generation in accretion disks. At Princeton, he won the Charlotte Elizabeth Procter Fellowship (2014) for his graduate research. He moved to Caltech in late 2015, where he is currently a Walter Burke Fellow in Theoretical Astrophysics. 